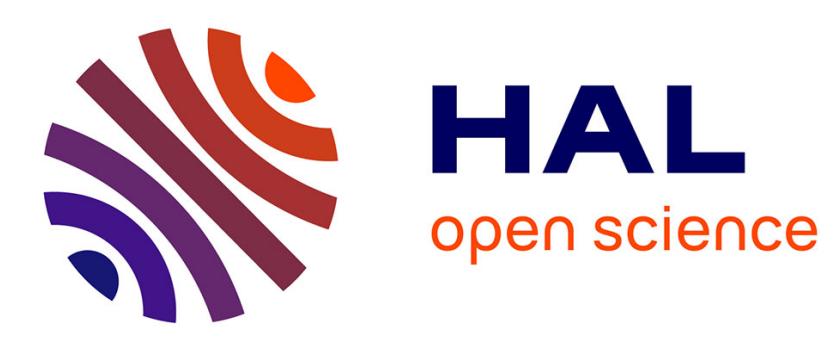

\title{
Filtering and Uncertainty Propagation Methods for Model-Based Prognosis of Fatigue Crack Growth in Unidirectional Fiber-Reinforced Composites
}

Elinirina I. Robinson, Julien Marzat, Tarek Raïssi

\section{- To cite this version:}

Elinirina I. Robinson, Julien Marzat, Tarek Raïssi. Filtering and Uncertainty Propagation Methods for Model-Based Prognosis of Fatigue Crack Growth in Unidirectional Fiber-Reinforced Composites. ASCE-ASME Journal of Risk and Uncertainty in Engineering Systems, Part A: Civil Engineering, 2018, 4 (4), pp.1-13. 10.1061/AJRUA6.0000991 . hal-01883533

\section{HAL Id: hal-01883533 \\ https://hal.science/hal-01883533}

Submitted on 28 Sep 2018

HAL is a multi-disciplinary open access archive for the deposit and dissemination of scientific research documents, whether they are published or not. The documents may come from teaching and research institutions in France or abroad, or from public or private research centers.
L'archive ouverte pluridisciplinaire $\mathbf{H A L}$, est destinée au dépôt et à la diffusion de documents scientifiques de niveau recherche, publiés ou non, émanant des établissements d'enseignement et de recherche français ou étrangers, des laboratoires publics ou privés. 


\title{
Filtering and uncertainty propagation methods for model-based prognosis of fatigue crack growth in unidirectional fiber-reinforced composites
}

\author{
Elinirina I. Robinson ${ }^{1}$ Julien Marzat $^{2}$, and Tarek Raïssi ${ }^{3}$.
}

\begin{abstract}
Prognosis aims at calculating the remaining useful life (RUL) of a system by estimating its current health state and then predicting its future behavior. In this paper, the prediction of fatigue crack growth in structural elements made of unidirectional fiber-reinforced composites is considered. Model uncertainty and measurement uncertainty are included, but future loading uncertainty is taken into account as well. Both cases of constant amplitude loading and variable amplitude loading (block loading) are treated. The analytical model that describes the fatigue crack growth is highly nonlinear and contains fixed model parameters that depend on material and loading parameters that may vary or not depending on the applied load. Thus, because of its ability to handle uncertainties, high nonlinearities and to perform joint parameter-state estimation, a particle filter is used. In a first part, fatigue crack growth prognosis under constant amplitude loading is realized. The loading parameters are constant and known a priori, while the model parameters are jointly estimated along with the crack length. Then, in a second part, fatigue crack growth prognosis under variable amplitude loading is performed. This time, the loading parameters are unknown and change abruptly at unknown time steps in accordance with the applied variable block loading. A two-sided cumulative sum (CUSUM) algorithm is implemented to detect abrupt load variations and help the particle filter to adapt and learn new loading parameters values. With the combination of these two techniques, the prognosis module could be informed of the sudden crack length increase, and will correct the predicted remaining useful life. In both case studies, real data from

\footnotetext{
${ }^{1} \mathrm{PhD}$ Student, ONERA/DTIS, Université Paris-Saclay, F-91123 Palaiseau, France (corresponding author). E-mail: elinirina.robinson@onera.fr

${ }^{2}$ Researcher, ONERA/DTIS, Université Paris-Saclay, F-91123 Palaiseau, France. E-mail: julien.marzat@onera.fr

${ }^{3}$ Professor, Conservatoire National des Arts et Métiers (CNAM), 75141 Paris, France. E-mail: tarek.raissi@cnam.fr
} 
fatigue tests on unidirectional fiber-reinforced titanium matrix composites is used.

Keywords: Model-based prognosis, particle filter, uncertainty propagation, fatigue crack growth, fiber bridging, composite materials, variable amplitude loading.

\section{INTRODUCTION}

Critical systems such as an aircraft or a spacecraft are made of complex components whose malfunction and failure could have unacceptable impacts on the users safety, the mission success and the costs related to maintenance operations. To address these safety and cost issues, a prognosis module should be integrated to these systems in order to continuously assess their state of health and estimate their remaining useful life (RUL). There are various prognosis approaches, but the most common classification divides them into three main categories (Liu et al. 2009): knowledgebased prognosis, data-driven prognosis and model-based prognosis. Each of these approaches has its advantages and drawbacks, and the choice of the method to use depends on the application domain and the information available about the system. The knowledge-based approaches (Biagetti 2004) make use of degradation rules that have been developed and refined by experts based on historical and empirical failure data. These kind of methods are easy to implement, but frequent updates are needed as new forms of faults that are not yet listed can occur. In data-driven approaches (Si et al. 2011), features from operating data such as current, temperature, or vibration signals are extracted, then statistical and machine learning techniques are employed to estimate and forecast the evolution of the degradation state. Data-driven approaches have the ability to transform highdimensional noisy data into lower-dimensional information for prognosis decisions. However, they are highly-dependent on the quantity and quality of operational data and therefore require a significant storage space. The third category gathers the model-based prognosis approaches (Luo et al. 2003) where mathematical models of system behavior and degradation evolution are used. Although these approaches can be difficult to set up since an accurate degradation model is seldom available, they can outperform knowledge-based and data-driven methods. Indeed, the capacity of model-based techniques to adapt the model to the evolution of the system degradation ensures an accurate prognosis if more information on the degradation become available. In this paper, 
emphasis is placed on the model-based prognosis techniques. The main challenge while developing a prognosis method is to include a measure of the uncertainty associated to predicted RUL values in order to enable risk-based decisions (Baraldi et al. 2013), (Sankararaman and Goebel 2013). Therefore, greater attention has been paid to the integration of uncertainty quantification in prognosis algorithms (Orchard et al. 2008). Uncertainty quantification consists in finding the different uncertainty sources, incorporating them into the algorithms, and finally propagating them to quantify uncertainty in the predicted RUL. The main types of uncertainty that inevitably influence RUL prediction are measurement uncertainty, modeling uncertainty and future loading uncertainty (Gu et al. 2007). Measurement uncertainty is due to sensor inaccuracy, modeling uncertainty is characterized by the difference between the degradation model and its real behavior, and future loading uncertainty is caused by various environmental factors that could possibly affect the evolution of the degradation. Usually, model-based prognosis methods represent the uncertainty in a probabilistic framework (Saha and Goebel 2008).

Many model-based prognosis methods that take uncertainty into account have been developed in the literature and were applied to various problems. For instance, (Daigle and Goebel 2011) have applied a particle filtering-based prognosis approach for pneumatic valves, in (Fan et al. 2015) the particle filter algorithm was implemented to predict the lumen maintenance life of LED light sources. A method combining relevance vector machine (RVM) and particle filtering was used for battery health prognosis in (Saha et al. 2009). In this paper, the focus is placed on an application of particular interest which is the crack growth prognosis. Indeed, this is a common issue for critical systems as fatigue damage occurs when a structure is subject to repeatedly applied loads. Even if the applied stress values are relatively low, the fact that they are cyclically applied tends to weaken the material and cause its failure. In order to avoid catastrophic events, the crack propagation must be carefully monitored in order to constantly evaluate the remaining useful life of the considered structure. In the literature, the problem of crack growth prognosis was treated for different models and with various methods. A hybrid data-driven and model-based approach based on RVM and model fitting was implemented in (Zio and Di Maio 2012) and applied to an academic fatigue 
crack growth process based on the Paris' law. Using the same test case, the crack growth prognosis was solved with a particle-filter based algorithm in (Zio and Peloni 2011). (Corbetta et al. 2015) proposed a particle filter sequentially updated via a Metropolis-Hastings (MH) algorithm for crack growth prognosis on helicopter fuselage panels. A machine learning approach based on artificial neural networks was used to estimate the stress intensity factor (SIF) range, which is required to calculate the crack growth at each cycle. In (Zárate et al. 2012), the SIF range was modeled by a polynomial equation with stochastic coefficients that were computed through Bayesian inference. The future crack length was then predicted using a Markov Chain Monte Carlo algorithm. The ability of these methods to predict the RUL of components subject to fatigue crack growth under measurement and modeling uncertainty has been proved through numerical examples. However, they have assumed known values of current and future loading, which is not the case in real life scenario.

In order to address the estimation of the loading amplitude, other researchers have introduced crack growth prognosis methods based on structural health monitoring data. The main idea is to use real-time monitoring data to build models that characterize fatigue loading history, and then based on these models, the future loading can be predicted. In (Ling and Mahadevan 2012), both flight parameters data related to acceleration and mass and data recorded from strain gauges were used to estimate and predict loading sequence through an autoregressive integrated moving average (ARIMA) modeling method and a Bayesian approach for the update process. The evaluation of the SIF range required for the crack growth calculation was made with a Gaussian process surrogate model that was previously trained with data from a finite element analysis. In (Pais and Kim 2015), usage monitoring data from an aircraft (acceleration, airspeed, angle of attack, fuel quantity and Mach number) were converted into a stress time history which was then transformed into a cyclic stress history via a rain-flow counting algorithm. The resulting cyclic stress history was used to consider the effects of variable amplitude loading in the determination of the crack growth direction. However, it was suggested that it could be used as the input into a prognosis method. What these proposed methodologies have in common is that real-time monitoring data related to 
the loading or flight parameters are required to build a model that characterizes the stress history. Moreover, a finite element model was needed for the calculation of the SIF range or to train its surrogate model. The main drawback of such an approach is that the learning phase can be time consuming and a significant amount of data is required.

In this paper, fatigue crack growth prognosis in fiber-reinforced titanium matrix composites based on real data from fatigue tests performed at ONERA/DMAS is performed. The considered real test case is an interesting application as fiber reinforced composites are gaining importance for use in structural elements. Indeed, the components last longer because composite materials have a high strength and are corrosion-resistant. The reader may refer to (Awad et al. 2012) to have more details about the use of fiber composite structures in civil engineering. In the literature, fatigue damage prognosis in composite materials with a known value of the applied stress was treated in (Chiachio et al. 2013). As composites where a densification of micro-cracks was observed under fatigue loadings were under study, the calculation of the SIF range was not suitable anymore and the micro-cracking energy release rate range $\Delta G$ was used instead. A modified Paris'law relating $\Delta G$ and the crack density $\rho$ was considered and the goal was to predict the saturation time of matrix micro-cracks and the onset of the consequent damage modes using a particle filter. The proposed methodology was applied to carbon-fiber-reinforced polymer (CFRP) cross-ply laminates and lamb wave signals recorded with piezoelectric sensors were used to estimate internal micro-crack density.

However, in the present work, fatigue crack growth prognosis in composite materials with long unidirectional fibers subjected to Mode I loading is examined. In such a case, a long matrix crack propagates perpendicular to the fiber direction (Movchan and Willis 1996), and the SIF range $\Delta K$ is used to characterize the evolution of the crack length. It is assumed that only crack length measurements are available, and no finite element model is used. Indeed, the effort was focused on the derivation of an analytical model of the SIF range. The obtained model contains model parameters that depend on material properties but also loading parameters that depend on external applied loads. Therefore, fatigue crack growth prognosis under known value of applied load is 
first considered in order to estimate the model parameters. For this purpose, a joint parameter-state estimation is performed with a particle filter because of its ability to handle uncertainties and the high nonlinearities of the SIF range model. The use of an extended Kalman filter (EKF) to solve the same fatigue crack growth prognosis problem was also investigated as it is easy to implement and requires lower computational time (Wang et al. 2017). The results were compared to those obtained with the particle filter using prognosis metrics. In a second study, the problem of fatigue crack growth prognosis under unknown constant and then unknown variable amplitude loading is addressed. Variable amplitude loading can be divided into three categories (Laseure et al. 2015): random loading, block loading and simple loading. In this work, block loading is considered, which means that loading parameters vary stepwise in time. Thus, using the model parameters estimated in the first study, the joint estimation of the crack length and of the unknown loading parameters was performed with a particle filter. However, if a significant abrupt load variation happens, the particle filter is not able to track the abrupt change in loading parameters values. To solve this problem, a detection algorithm, namely the two-sided cumulative sum (CUSUM), is implemented in parallel with the particle filter to catch any sudden deviation of loading parameters. This way, the particle filter will receive the information and then reinitialize the previous estimates in order to learn the new loading parameters values. This detection step enables to adapt the prognosis algorithm to the changes in crack length evolution and therefore give a more accurate prediction of the RUL. Indeed, an increase in the amplitude loading can significantly accelerate the crack growth rate and lead to the critical crack length causing the failure of the component. In both studies, real crack length trajectories were used, and the prognosis metrics that were used to validate the ability of the particle filter to perform fatigue crack growth prognosis in such materials are accuracy, precision and timeliness.

This paper is organized as follows. Section 2 introduces the general principle of fatigue crack growth model-based prognosis and the model that describes fatigue crack propagation in unidirectional fiber-reinforced composite materials. In Section 3, the joint parameter-state estimation methodology with the particle filter is presented, as well as the two-sided CUSUM algorithm. 
Then, numerical results which illustrate the efficiency of particle filtering prognosis on real fatigue crack growth data are reported in Section 4, where a first part concerns the case of unknown model parameters under known external loading amplitude, whereas in the second part the external loadings considered as unknown. Finally, a general conclusion about fatigue crack growth prognosis in unidirectional fiber-reinforced composites and perspectives for future work are given in Section 5.

\section{PROBLEM STATEMENT}

The central idea of model-based prognosis is to use a dynamic mathematical model that describes the evolution of a degradation occurring in a system or a component. In most cases, as the degradation state cannot be observed directly, measurements from different sensors can be used to collect information about it. In the application considered in this paper, the degradation in question is the crack length and the collected data are crack length measurements. Usually, due to implementation constraints, a discrete-time state-space representation is employed to relate the mathematical model of the degradation to the data from the different sensors to determine the evolution of the degradation state at any time instant:

$$
\begin{aligned}
& \mathbf{x}_{k}=f\left(\mathbf{x}_{k-1}, \theta_{k-1}, \mathbf{u}_{k-1}, \mathbf{w}_{k-1}\right) \\
& \mathbf{y}_{k}=h\left(\mathbf{x}_{k}, \theta_{k}, \mathbf{u}_{k}, \mathbf{v}_{k}\right)
\end{aligned}
$$

where $\mathbf{x} \in \mathbb{R}^{n_{x}}$ denotes the state vector, $\theta \in \mathbb{R}^{n_{\theta}}$ represents the parameter vector, $\mathbf{y} \in \mathbb{R}^{n_{y}}$ is the measured outputs, $\mathbf{u} \in \mathbb{R}^{n_{u}}$ is the vector of system inputs and $k \in \mathbb{N}$ is a discrete time step. The functions $f$ and $h$ describe respectively the evolution of the state and the measurements over time, and there is no restrictions on their functional forms. The variables $\mathbf{w}$ and $\mathbf{v}$ are respectively the process and measurement noises which represent the model and measurement uncertainties.

The fatigue crack growth model-based prognosis process is based on three steps: (i) current crack length estimation, (ii) future crack length prediction and (iii) RUL calculation. The first step is a filtering problem since the current crack length is estimated using measurements from sensors. This estimation is performed as long as data is collected, i.e until the prediction time denoted by 
$k_{p}$. Then, from this time instant, the second step involves the forecasting of the future crack length for time instants $k>k_{p}$ without new measurements. The future crack length is predicted until the failure threshold is reached, giving the predicted failure time $k_{f}$. Finally, in the last step of prognosis, the RUL at time $k_{p}$ which is denoted by $R U L\left(k_{p}\right)$ can be calculated as:

$$
R U L\left(k_{p}\right)=k_{f}-k_{p} .
$$

However, as the predicted crack length is uncertain, the predicted failure time $k_{f}$ is also uncertain, making the RUL a random variable. Therefore, prognosis methods should account for the different uncertainty sources that affect the estimation of the current and future crack length. In that way, propagating these uncertainties through the degradation model will allow to obtain the subsequent RUL probability density function (pdf).

In this paper, the problem of fatigue crack growth prognosis in fiber-reinforced titanium matrix composite materials is addressed. This will allow to continuously assess the RUL of a structure made of such composite material in which a fatigue crack growth has occurred. As a modelbased prognosis approach is used, the first requirement is to establish an analytical model to assess dynamic crack propagation in such materials. Before introducing this model, the experimental procedure to collect the real crack growth data used in this work is described.

\section{Material and experimental procedures}

A composite material is the combination of two or more different materials in order to create a superior material with different properties (stronger, lighter, etc.). Composites are mainly made up of two constituent materials: matrix and reinforcement. There are three main kinds of materials that are used for the matrix (polymer, metal and ceramic) and also different forms of reinforcement material (particles, fibers or laminates). In this work, the proposed methodology is applied to fatigue crack growth prognosis in titanium metal matrix composites with silicon carbide fibers used as reinforcement materials. The fatigue test data were previously used in (Maire et al. 2000) to establish and validate a model to describe the fatigue crack growth in the concerned specimens, 
but the problem of RUL prognosis was not addressed. For consistency, the experimental protocol is summarized in what follows.

The titanium-matrix composite studied was SCS-6/Ti-6242. Ti-6242 is a near alpha titanium alloy with the composition Ti-6Al-2Sn-4ZR-2Mo (percent by weight), and this matrix was reinforced with $140 \mu \mathrm{m}$ diameter SCS-6 Textron fibers. The fibers are regularly spaced in the matrix in such a way as to obtain a unidirectional composite. A parallelepiped notched specimen (Fig. 1) with a nominal width of $8 \mathrm{~mm}$, thickness of $2.1 \mathrm{~mm}$ and length of $160 \mathrm{~mm}$ was machined from the composite material in a manner that the length of the specimen is parallel to fiber axis. An elliptic notch was drilled in the middle of the specimen in order to initiate the crack growth.

During the experiment, crack growth trajectories of 8 samples of different notch lengths were recorded. Some specimens were tested under constant amplitude loading, while the others were subjected to load variations. For every specimen, a uniaxial cyclic loading oriented along the fiber direction was applied. The stress ratio $R=\sigma_{\min } / \sigma_{\max }$ was equal to 0.1 . The fatigue tests were performed at room temperature and at $400^{\circ} \mathrm{C}$ under a frequency of $50 \mathrm{~Hz}$. In order to measure the crack length, photographs of the crack extension were recorded by a digital camera monitored by a computer. The reader may refer to (Thurner 2015) to be more informed about the real-time detection and measurement of cracks. The typical cracking geometry involved the propagation of a crack in the matrix on each side of the notch, propagating perpendicularly to the loading direction (Fig. 2).

The crack propagated through all the thickness of the specimens and across their entire width without causing the rupture of the composite material. This phenomena is due to unbroken fibers that have bridged the matrix crack. Indeed, as the fiber stress level did not exceed the value of the fiber strength, no fiber broke during the experiments. The constraints of these bridging fibers can be modeled by the distribution of a closure pressure $P$ acting in the direction opposite to the applied stress $\sigma_{a}$ in the bridged zone (Fig. 3). In this case, the bridged zone is equivalent to the crack length minus the notch length $2 a_{0}$. 


\section{Description of the crack propagation model}

It is assumed that the composite has a linear elastic behavior except in a very small region at the crack tip, therefore the principles of linear elastic fracture mechanics can be applied. The following modified Paris' law that was previously used in (Maire et al. 2000) to study the same fatigue test dataset can then be used to model the Mode I crack propagation:

$$
\frac{d a}{d N}=C\left(\Delta K_{m}-K_{t h}\right)^{m}
$$

where $a$ is the crack size, $N$ is the number of cycles. Since the crack growth observed in the composite material was limited to the matrix cracking, the effective crack-driving force is assumed to be the SIF range $\Delta K_{m}$ experienced by the matrix. The constants $C, m$ and $K_{t h}$ depend on matrix properties. $K_{t h}$ is the threshold value of the SIF below which no crack growth occurs.

The calculation of the SIF range $\Delta K_{m}$ is the main challenge in this modeling stage. A simple expression of the SIF range can be available. However, in more complex structures as composite materials, an analytical closed form of the SIF does not always exist or is too complicated to establish. In these cases, finite element simulations can be run to calculate the SIF values associated to different crack lengths. Then, based on the obtained database of SIFs, a regression model is used to allow the evaluation of the SIF for any crack length. This technique was used in (Corbetta et al. 2015) where a machine learning approach based on artificial neural networks was used to provide estimates of SIFs. In (Neerukatti et al. 2014), two regression techniques were used, namely least absolute shrinkage and selection operator (LASSO) and relevance vector machine. Although very efficient to obtain good estimations of the SIF, the main drawback of such an approach is that the learning stage can be time consuming and a significant amount of data is required. In this work, an analytical expression of the stress intensity factor for bridged cracks in composite materials was established, based on different studies found in (Johnson et al. 1996).

The first step to determine $\Delta K_{m}$ is to relate it to the continuum SIF range $\Delta K_{t i p}$ which is the homogenized composite stress intensity factor. Many discrete-continuum relationships were pro- 
posed, and three of them were compared in (Bakuckas and Johnson 1993). In their study, the one that gave the best results in the modeling of fiber-bridging effect on $\Delta K_{m}$ was established by (McMeeking and Evans 1990):

$$
\Delta K_{m}=\Delta K_{t i p}
$$

In order to calculate $\Delta K_{t i p}$, the weight function technique proposed by (Bueckner 1970) that allows to calculate a stress intensity factor for arbitrary stress distributions is applied. In this case study, the applied stress range on the crack surface is $\Delta \sigma_{b r}=\Delta \sigma_{a}-\Delta P(x)$, therefore we have:

$$
\Delta K_{\text {tip }}=2 \int_{0}^{a}\left(\Delta \sigma_{a}-\Delta P(x)\right) G(x, a, c) \mathrm{d} x
$$

where $G(x, a, w)$ is a weight function that depends on the geometry of the specimen, $c$ is the specimen width and $2 a$ is the crack length.

For a center crack configuration in a finite width specimen, the following expression of $\Delta K_{t i p}$ is finally obtained (Zheng and Ghonem 1996):

$$
\Delta K_{t i p}=F \Delta \sigma_{a} \sqrt{\pi a}-2 F \sqrt{\frac{a}{\pi}} \int_{a_{0}}^{a} \frac{\Delta P(x)}{\sqrt{a^{2}-x^{2}}} \mathrm{~d} x
$$

where $F$ is a geometric factor that depends on the specimen width (various expressions can be found in (Tada et al. 1973)), $2 a_{0}$ is the length of the unbridged zone (i.e. notch length) and $x$ is the distance from the crack center. It can be noted that this expression of $\Delta K_{t i p}$ takes into account both the contribution of the applied remote stress $\Delta \sigma_{a}$ and the contribution of the bridging stress $\Delta \sigma_{b r}$ produced by unbroken fibers in the crack wake.

Finally, the model that describes crack growth propagation in the studied specimens is given by:

$$
\frac{d a}{d N}=C\left(F \Delta \sigma_{a} \sqrt{\pi a}-2 F \sqrt{\frac{a}{\pi}} \int_{a_{0}}^{a} \frac{\Delta P(x)}{\sqrt{a^{2}-x^{2}}} \mathrm{~d} x-K_{t h}\right)^{m}
$$

The determination of the change in closure pressure $\Delta P(x)$ is the critical issue of the fatigue crack propagation problem in fiber reinforced composites. In the literature, two types of analytical 
models have been widely used to calculate $\Delta P(x)$ : (i) the shear-lag model and (ii) the fiber pressure model. For more details about these two approaches, the reader may refer to (Ghosn et al. 1992).

As an alternative to these methods, (Davidson 1992) have used a uniform closure pressure over the entire bridged zone and obtained satisfying results for SCS-6/Ti-6Al-4V composite materials. This approach was considered in this paper as it allows to avoid the integration of $\Delta P(x)$ to calculate $\Delta K_{t i p}$, which highly decreases the computational time. Indeed, this is an important criteria for online RUL prognosis.

Adopting this constant approximation of the closure pressure leads to the following model:

$$
\frac{d a}{d N}=C\left(F \Delta \sigma_{a} \sqrt{\pi a}-2 F \sqrt{\frac{a}{\pi}} \Delta P \int_{a_{0}}^{a} \frac{1}{\sqrt{a^{2}-x^{2}}} \mathrm{~d} x-K_{t h}\right)^{m}
$$

where

$$
\int_{a_{0}}^{a} \frac{1}{\sqrt{a^{2}-x^{2}}} \mathrm{~d} x=\frac{\pi}{2}-\arcsin \left(\frac{a_{0}}{a}\right)
$$

Finally, the model that is used for crack growth prognosis in the studied fiber-reinforced titanium matrix composite material is:

$$
\frac{d a}{d N}=C\left(F \Delta \sigma_{a} \sqrt{\pi a}-2 F \sqrt{\frac{a}{\pi}} \Delta P\left(\frac{\pi}{2}-\arcsin \left(\frac{a_{0}}{a}\right)\right)-K_{t h}\right)^{m}
$$

The model (11) that was obtained involves two different types of parameters. One needs to distinguish between model parameters and loading parameters:

- $m$ and $C$ are constant model parameters which depend on the material under study and various testing conditions (stress ratio $R$, temperature, etc.). They can be estimated for a given specimen and considered to have the same values for the other similar test specimens;

- $\Delta \sigma_{a}$ and $\Delta P$ are loading parameters directly related to the external loading applied to each specimen. Their values may therefore vary according to the loading conditions. The estimation of these two loading parameters is an interesting feature as most of the existing prognosis methods have assumed known values of current and future loading conditions, 
which is not the case in real life scenarios. Their real-time monitoring would allow the prognosis system to catch any abrupt load variation and update the RUL estimation. Indeed, depending on the load variation suffered by the structure, the crack growth rate may rapidly increase and cause its sudden failure.

Because of their different nature (constant ore time-varying), these two sets of parameters will not be estimated at the same time for the fatigue crack prognosis in the studied composite material. Firstly, a dataset of crack growth trajectory from a specimen tested under a known constant amplitude loading will be used to estimate the model parameters $m$ and $C$ and perform fatigue crack growth prognosis. Then, using these estimated values of $m$ and $C$, the case of fatigue crack growth prognosis under variable amplitude loading where $\Delta \sigma_{a}$ and $\Delta P$ are unknown will be considered. In both studies, the parameters are jointly estimated along with the crack length with a particle filter. However, in the second case study, $\Delta \sigma_{a}$ and $\Delta P$ are time-varying parameters that change abruptly with the applied load. A CUSUM algorithm is then used to detect load variations and help the particle filter to adapt and learn the new loading parameters values.

In the next section, the general methodology for joint estimation of the parameters along with the crack length using a particle filter is first presented. Then, it will be explained how the particle filter and a detection algorithm can be combined to correctly estimate the crack length in a structure subjected to variable amplitude loading.

\section{FATIGUE CRACK GROWTH PROGNOSIS WITH A PARTICLE FILTER COMBINED WITH A TWO-SIDED CUSUM ALGORITHM}

A particle filter was chosen to perform the joint estimation of the unknown parameters along with the crack length in order to calculate the RUL of the composite specimens subjected to fatigue crack growth. The ability of the particle filter to perform uncertainty propagation but also to deal with the high nonlinearities of the fatigue crack growth analytical model (11) has motivated this choice. Moreover, as the particle filter is a Bayesian filtering method, the RUL predictions are updated and improved as new crack length data is collected. 
When specimens were tested under variable amplitude loading (block loading), loading parameters are piecewise constant functions of time. In order to detect load variations and to keep an accurate estimate of these loading parameters, a two-sided CUSUM algorithm is added to the particle filter so it can keep on tracking the time-varying parameters. The idea is to jointly estimate the unknown loading parameters along with the crack length using the particle filter, while monitoring their estimations with the two-sided CUSUM algorithm to detect abrupt load variations. If a load variation happens, the CUSUM algorithm informs the particle filter which has then to reinitialize its previous estimates of the loading parameters and learn their new values.

In this section, the sequential importance resampling (SIR) particle filter is first introduced briefly, then the two-sided CUSUM algorithm is presented, and finally model-based prognosis process under unknown loading parameters that uses the combination of these two techniques is described.

\section{Particle filter for joint parameter-state estimation}

Particle filtering allows to recursively estimate and update the pdf of the state vector $\mathbf{x}_{k}$ based on the discrete state-space system characterized by equations (1) and (2). In this work, the unknown model parameters denoted by the vector $\theta \in \mathbb{R}^{n_{\theta}}$ are included in the state vector $\mathbf{x} \in \mathbb{R}^{n_{x}}$. This allows to form an augmented state vector $\mathbf{X}=\left[\begin{array}{ll}\mathbf{x} & \theta\end{array}\right]$ in order to perform the identification of the unknown parameters in conjunction with state estimation using the particle filter. In this paper, $x$ is the crack length while $\theta$ contains the model parameters $m$ and $C$ or the loading parameters $\Delta \sigma_{a}$ and $\Delta P$ depending on the considered case study.

In the particle filter approach, the state pdf at time instant $k$ is approximated by a set of $N_{\text {part }}$ particles $\left\{X_{k}^{i}\right\}_{i=1}^{N_{\text {part }}}$ representing points in the state space, and a set of associated weights $\left\{\omega_{k}^{i}\right\}_{i=1}^{N_{\text {part }}}$ denoting discrete probability masses:

$$
p\left(X_{k} \mid y_{0: k}\right) \approx \sum_{i=1}^{N_{\text {part }}} \omega_{k}^{i} \delta\left(X_{k}-X_{k}^{i}\right) \text { with } \sum_{i=1}^{N_{\text {part }}} \omega_{k}^{i}=1
$$

where $\delta$ is the Dirac delta function. 
A sequential importance resampling (SIR) particle filter is used in this work. The reader may refer to (Arulampalam et al. 2002) to have more details about the various particle filter algorithms and the SIR particle filter that will be briefly presented in what follows. It is based on three main steps that are prediction, update and resampling:

Initialization

- Draw particles $X_{0}^{i} \sim p\left(X_{0}\right)$

- Compute the initial weights $\omega_{k}^{i}=\frac{1}{N_{\text {part }}}$

1. Prediction

- Simulate the state equation (1) to generate a new set of $N_{p}$ particles $X_{k}^{i=1: N_{p a r t}}$ which are realizations of the predicted pdf $p\left(X_{k} \mid y_{0: k-1}\right)$.

2. Update

- Each sampled particle is assigned a weight based on the likelihood $p\left(y_{k} \mid X_{k}\right)$ :

$$
\omega_{k}^{i}=\omega_{k-1}^{i} p\left(y_{k} \mid X_{k-1}^{i}\right)=\omega_{k-1}^{i} \frac{p\left(y_{k} \mid X_{k}^{i}\right) p\left(X_{k}^{i} \mid X_{k-1}^{i}\right)}{p\left(X_{k}^{i} \mid X_{k-1}^{i}, y_{k}\right)}
$$

- Normalize the weights:

$$
\omega_{k}^{i}=\omega_{k}^{i}\left(\sum_{i=1}^{N_{\text {part }}} \omega_{k}^{i}\right)^{-1}
$$

3. Resampling

- Degeneracy problem: the weight variance increases and after a few iterations all but one particle may have a negligible weight (Daum 2005). Particles with small weights are eliminated so that the computational efforts are concentrated in those having large ones.

- Resampling condition: if the effective sample size $N_{\text {eff }}$ is under some threshold 
$N_{t h}$, a re-sampling procedure is done. An estimate of $N_{e f f}$ is

$$
\hat{N}_{e f f}=\left(\sum_{i=1}^{N_{\text {part }}}\left(\omega_{k}^{i}\right)^{2}\right)^{-1}
$$

- Using the inverse cumulative distribution function method (Arulampalam et al. 2002) and the current set $\left\{X_{k}\right\}_{i=1}^{N_{p a r t}}$, a new set $\left\{\tilde{X}_{k}\right\}_{i=1}^{N_{\text {part }}}$ is drawn to replace the current one. Finally, with $\tilde{\omega}_{k}^{i}=N_{\text {part }}^{-1}$, the state is given by:

$$
\hat{X}_{k}^{i}=\sum_{i=1}^{N_{\text {part }}} \tilde{\omega}_{k}^{i} \tilde{X}_{k}^{i}
$$

This classical SIR particle filter algorithm is applied during the first step of the prognosis process which consists in estimating the current augmented state vector using data from the different sensors. This step is realized as long as measurements are available until the prediction time $k_{p}$ from which a prediction of the future augmented state vector is performed. During the forecasting step, no more measurements are collected. However, the update of the particle weights depends on the acquisition of new measurements. To overcome this difficulty, the state is propagated only using the state model (1) while the particle weights are propagated in time without any changes. In other words, only the prediction step is repeated until the chosen failure threshold is reached. Considering that the particle weights are invariant for time instants $k>k_{p}$ leads to a negligible approximation error with respect to other sources of error such as wrong choices of noise parameters or model inaccuracies (Orchard and Vachtsevanos 2007).

The general methodology that was presented above is adopted when the constant model parameters $m$ and $C$ are jointly estimated along with the crack length. In the case where the crack is propagating under unknown variable amplitude loading (block loading), the loading parameters are unknown and time-varying (piecewise constant functions of time). However, the SIR particle filter itself cannot handle sudden change in loading parameters. Indeed, between two load variations, the loading parameters have constant values. The marginal posterior parameter distributions 
become increasingly concentrated around the true parameters values as more data is available. Thus, if some abrupt load variation occurs, the new loading parameters values may fall far into the tails of the parameter distributions, leading to particles with negligible weights. The particle filter is not able to estimate the new parameters values and a reinitialization of the particle system is then needed. In this work, a two-sided CUSUM algorithm is implemented to detect load variations and trigger the re-initialization of the particle filter for the learning of the new loading parameters values.

\section{Two-sided CUSUM algorithm}

A two-sided CUSUM algorithm is used to detect load variations during experiments. It is the combination of two CUSUM algorithms, one for the detection of an increase in the mean of the monitored variable, and the other one to detect a decrease in the mean. The general idea is to calculate a cumulative sum $S_{k}$ that depends on the monitored process $\Delta x$, on its initial mean value $\mu_{0}$ and on the minimal size of change to detect denoted by $v$. And when the value of the sum exceeds a predefined threshold value $S_{t h}$, a change in the mean value is detected. Therefore, the two-sided CUSUM algorithm is based on the following equations:

$$
\begin{cases}S_{k}^{+} & =\max \left(0, S_{k-1}^{+}+\Delta x_{k}-\mu_{0}-\frac{v}{2}\right) \\ S_{k}^{-} & =\max \left(0, S_{k-1}^{-}-\Delta x_{k}-\mu_{0}-\frac{v}{2}\right) \\ N_{\text {detect }} & =\min \left\{k: S_{k}^{+} \geq S_{t h} \cup S_{k}^{-} \geq S_{t h}\right\}\end{cases}
$$

where $N_{\text {detect }}$ is the time at which the detection is made. There are two parameters that have to be chosen in this algorithm: $S_{t h}$ and $v$. This choice depends on how the signal to process looks like, and for a Gaussian distribution, one can set $S_{t h}=2 \frac{\sigma_{\Delta x}}{v}$ where $\sigma_{\Delta x}$ is the standard deviation of $\Delta x$. Further information about the two-sided CUSUM algorithm can be found in (Blanke et al. 2006). 


\section{Prognosis methodology with the particle filter and the detection algorithm}

The constant monitoring of loading parameters with the detection algorithm allows not only to detect abrupt load variations but also to reinitialize the parameters values right after the alarm. This would help the particle filter to converge more quickly and more easily to the actual crack length. This procedure leads to the introduction of a new uncertain parameter which is the initial loading parameters values after load variation. In most cases this value is unknown, but the user may have an order of magnitude of it. Indeed, depending on the monitored system, the critical input value that can accelerate the degradation state evolution can be obtained from expert knowledge. Therefore, because of the uncertainty associated to this value, it is included in an interval $L=[\underline{L}, \bar{L}]$.

\section{NUMERICAL APPLICATIONS}

The aim of this section is to show how online fatigue crack growth prognosis with a SIR particle filter can be assessed in fiber-reinforced titanium matrix composite materials. The case of a fatigue crack prognosis with unknown model parameters $m$ and $C$ but known loading parameters $\Delta \sigma_{a}$ and $\Delta P$ is first presented. A dataset from a specimen tested under constant amplitude loading is used. In order to justify the choice of a particle filter rather than an extended Kalman filter (EKF) which is easier to implement and is less time consuming, the performance of these two stochastic filters will be compared. Then, in a second part, a more complex and realistic case of fatigue crack growth prognosis where the loading parameters $\Delta \sigma_{a}$ and $\Delta P$ are unknown is considered. In this second example, a dataset from a specimen tested under variable amplitude loading is used.

In order to evaluate the performance of the particle filter and of the EKF in estimating the RUL of the considered composite materials, three prognosis metrics (accuracy, precision and timeliness) are calculated. Accuracy measures the degree of closeness of the predicted RUL to the actual RUL, and its values are between 0 and 1 where 1 gives the best accuracy. Precision evaluates the narrowness of the interval in which the RUL predictions fall, and ranges between 0 and 1 which reflects the highest precision. Finally, timeliness indicates the relative position of the predicted RUL pdf along the time axis with respect to the occurrence of the actual failure event. There are three cases: (i) the failure occurs after the predicted failure time, (ii) the failure occurs at the 
same time as the predicted failure time, and finally, (iii) the failure occurs earlier than predicted. This last case must be absolutely avoided, that is why the timeliness function allows to penalize late predictions. Timeliness has positive values and 0 is the best score. More details about the formulation of these metrics can be found in (Robinson et al. 2016), where the SIR particle filter and the EKF were compared on synthetic data generated with a Paris' law to simulate a simple fatigue crack growth test case.

For both case studies, the input parameters required for the implementation of the algorithms are provided, then the simulation results are shown. Finally, the prognosis methods are compared using the performance metrics that have been defined above. The following discrete-time form of the crack growth model (11) is used:

$$
a_{k+1}=a_{k}+C \Delta N\left[F \Delta \sigma_{a_{k}} \sqrt{\pi a_{k}}-2 F \sqrt{\frac{a}{\pi}} \Delta P_{k}\left(\frac{\pi}{2}-\arcsin \left(\frac{a_{0}}{a_{k}}\right)\right)-K_{t h}\right]^{m}
$$

This model is randomized in order to take the different uncertainty sources into account. Modeling and measurement uncertainty are integrated by adding zero-mean Gaussian noises to the state and measurement equations, that are respectively $\mathbf{w}_{k}$ and $\mathbf{v}_{k}$. Moreover, the unknown model parameters to estimate in conjunction with the crack length are considered as Gaussian random variables.

\section{Unknown model parameters $m$ and $C$}

In this first case study, a crack growth trajectory dataset where the loading amplitude is maintained constant is used. The loading parameters $\Delta \sigma_{a}$ and $\Delta P$ are then constant and equal to 196 MPa. The objective here is to jointly estimate and forecast the crack length and the two unknown material parameters $m$ and $C$, therefore the augmented state vector is defined as $\mathbf{X}_{k}^{\top}=$ $\left[\begin{array}{lll}a_{k} & C_{k} & m_{k}\end{array}\right]$. The variances of the stochastic variables that were used for the implementation of the particle filter and of the EKF algorithms are listed in Table 1. In this work, filter tuning was performed offline on the basis of a trial and error procedure. The online tuning of the particle filter was not addressed in this paper as it is a complex problem when a highly nonlinear model and 
real data are both involved. In the literature, some references have treated this problem outside the context of prognosis. In (Kontoroupi and Smyth 2015), an online strategy to jointly estimate, through the formulation of an augmented vector, the state vector, the model parameters and the statistical parameters (covariance and mean) of the process and observation noises is proposed. They assume some conjugate prior distributions for both mean and covariance matrix of noises and perform Bayesian updating using an unscented Kalman filter (UKF). However, they point out the possible limitations of such a method due to the curse of dimensionality.

The values of the initial state vector have to be determined for the initialization of the filtering algorithms. The initial crack length $a_{0}$ is supposed to be known because the prognosis module is launched only if a crack growth is detected in the monitored component. In the tested specimen, this value is $a_{0}=3.1 \mathrm{~mm}$. Concerning $m$ and $C$, as they are material constants, their order of magnitude can be a priori known to initialize the algorithms. In this case, we have chosen $m_{0}=2.4$ and $\log (C)=-23.65$. Finally, measurements were collected every $\Delta N=1172$ cycles and a total number of 500 particles was used by the filter. In the figures presented in the next subsections, the plotted curves correspond to the mean estimation.

The crack growth prognosis has been done for different prediction time cycles $N_{p}$, which means that the number of available measurements in the observation interval has been increased over cycles, as in on-line prognosis. From prediction time cycle $N_{p}$, the forecasting of the future crack length was performed without any measurements until the threshold was reached in order to deduce the RUL value. This critical crack length was fixed at $7.5 \mathrm{~mm}$. In order to evaluate the performance of the algorithms in terms of accuracy, precision and timeliness, 100 experiments have been done to simulate experimental variability. Concerning the choice of the performance metrics parameters $\left[I_{\min }, I_{\max }\right]$ and $I_{0}$, they depend on the material and user requirements. They can be derived from prior knowledge about the fatigue behavior of the material under study characterized by its $S-N$ curve (Kawai and Itoh 2014). Based on these considerations, the user can define an allowable margin as a decision criterion. In this case, we have chosen $I_{0}=2 \times 10^{5}$ cycles. As for the interval $\left[I_{\text {min }}, I_{\text {max }}\right]$, its width equals $I_{0}=2 \times 10^{5}$ cycles. 
The evolution of the crack growth prognosis for different values of $N_{p}$ are shown in Fig. 4, Fig. 5 and Fig. 6 for the EKF and in Fig. 7, Fig. 8 and Fig. 9 for the PF. The performance metrics values for both methods are listed in Table 2. The results show that the EKF did not manage to predict correctly the future crack length until time cycle $N_{p}=2.794 \times 10^{5}$ cycles. Indeed, the EKF gives reliable results only if the system is locally linear and is not highly nonlinear, or when there is no joint parameter-state estimation as in (Wang et al. 2017). The only way to obtain satisfactory results with the EKF was to decrease the standard deviation of the exponent parameter $m$ to $\sigma_{m}=10^{-6}$, however it is not a realistic value because the parameter $m$ generally varies by $10 \%$ from one specimen to another. As regards the particle filter, even at only $N_{p}=1.198 \times 10^{5}$ cycles, the RUL prognosis gives appropriate results in terms of accuracy, precision and timeliness, and the metric values improve as more measurements are available.

The high performance of the particle filter for the fiber-bridged crack prognosis was highlighted in the results and confirms the results obtained in (Robinson et al. 2016) on an academic test case with a Paris' law. Moreover, the choice of the constant value of the closure pressure is appropriate in this case. Thus, the proposed model for fatigue crack growth propagation in fiber-reinforced composites can be validated.

\section{Unknown loading parameters $\Delta \sigma_{a}$ and $\Delta P$}

This second case study is based on a dataset where the cracked specimen was subjected to variable loading amplitude. A joint input-state estimation will allow the constant monitoring of external loads while estimating the crack length. The augmented state vector is then defined as $\mathbf{X}_{k}^{\top}=\left[\begin{array}{lll}a_{k} & \Delta \sigma_{a_{k}} & \Delta P_{k}\end{array}\right]$. The variances of these stochastic variables that were used for the implementation of the particle filter algorithm are listed in Table 3.

The initial crack length $a_{0}$ is supposed to be known because the prognosis module is launched only if a crack growth is detected in the monitored component. In the tested specimen, this value is $a_{0}=0.7 \mathrm{~mm}$. Concerning the inputs loading parameters $\Delta \sigma_{a}$ and $\Delta P$, their initial values are assumed to be included in the interval $L_{0}=[300,400] \mathrm{MPa}$.

In this part, the material parameters $m$ and $C$ are assumed to be constant variables as the focus 
is placed on the estimation of the unknown loading parameters. They have already been calculated in a previous work, and we have $m=2.4$ and $C=5.4 \times 10^{-11}$. Finally, a total number of 500 particles was used and crack length measurements were collected every $\Delta N=1172$ cycles.

\section{Fatigue crack growth under unknown constant amplitude loading}

The first step of prognosis consists in jointly estimating the current crack length $a$ and the unknown loading parameters $\Delta \sigma_{a}$ and $\Delta P$ using the crack growth model and the collected crack length data at each time step $k$ corresponding to a cycle number $N$. Then, from a prediction time cycle $N_{p}$, the forecasting of the future state vector is performed without any new measurements.

First of all, in order to demonstrate the robustness of the proposed particle filter to the initial unknown loading parameters values, the prognosis results for different initial values of $\Delta \sigma_{a}$ and $\Delta P$ in $L_{0}$ have been plotted in Fig. 10.

It can be seen that despite the uncertainty on the initial loading input values, the particle filter always manages to converge to the actual crack length. This is an important feature of the proposed joint input-state estimation algorithm because in real-time prognosis, this value is unknown.

Moreover, one can notice that the crack length does not increase and the estimated unknown loading parameters remain constant after the transient state (Fig. 11). Indeed, the crack growth stops after some extension because of the fiber bridging phenomenon mentioned in Section 2. Therefore, the crack growth reaches a steady-state and the failure threshold will not be reached.

In the following, a case of crack growth under variable amplitude loading is treated to study the ability of the proposed methodology to jointly estimate the crack length and the unknown loading parameters even in these circumstances.

\section{Fatigue crack growth under unknown variable amplitude loading}

In this subsection, crack growth prognosis under unknown variable amplitude loading is considered. The crack length measurements are from the real dataset obtained during fatigue test on fiber-reinforced titanium matrix composite materials presented in Section 2.

As a first step, the estimation of the crack length and the unknown loading parameters is performed using the available measurements. Then, the forecasting step is realized from the prediction 
time cycle $N_{p}$. Before load variation, the prognosis results are the same as described in the previous subsection. After load variation, the forecasting of the future crack growth without new measurements is made at different prediction time cycles $N_{p}$. The evolution of the future crack length predictions are depicted in Fig. 12 and Fig. 13. The first part of the crack length evolution is not shown as it is the same as in Fig. 10.

The higher amplitude of the external applied load has led to an increase in the crack length, which indicates that the critical stress has been attained. The results show that the particle filter has some difficulties to converge even after several time cycles of estimation using the measurements. Indeed, the particle filter needs more data in order to estimate the unknown loading parameters whose values have significantly increased after this abrupt load variation. This problem might be addressed by increasing the number of particles. In this study, 500 particles were used, and a test with 3000 particles was realized. The results were almost the same, and this number cannot be further increased because the computational time would be too important, which is not suitable for online applications.

In order to circumvent this issue, the two-sided CUSUM algorithm presented in Section 3 is integrated to the particle filter. The unknown loading parameters $\Delta \sigma_{a}$ and $\Delta P$ are constantly monitored to detect the sudden load variation. Indeed, as these inputs are related to the applied load, when the loading amplitude changes, their values change as well (Fig. 14). It can be seen that this monitoring must start after the transient state to avoid any false detection.

The parameter $\mu_{0}$ of the CUSUM algorithm is the mean value of the estimated parameters in the transient state. The minimal size of change to detect in the unknown loading parameters variables was xed to $v=2 \mathrm{MPa}$. This value was chosen based on the parameter estimations shown in Fig. 11. The two-sided CUSUM algorithm has detected the variation at $N_{\text {detect }}=10.881 \times 10^{6}$ cycles while the actual load variation time is $N_{\text {load }}=10.868 \times 10^{6}$ cycles.

After the detection of load variation, the values of $\Delta \sigma_{a}$ and $\Delta P$ are reinitialized. The choice of the interval $L$ is based on the a priori knowledge of the necessary load amplitude that may cause a rapid propagation of the crack in the considered specimen. The values $\Delta \sigma_{a_{c r i t}}$ and $\Delta P_{c r i t}$ associated 
to the critical load are known to be around $450 \mathrm{MPa}$. Therefore, in order to take the uncertainty associated to this value into account, it was considered that the reinitialized values of $\Delta \sigma_{a}$ and $\Delta P$ belong to the interval $L=[400,500]$. This has allowed to estimate and then forecast an interval that contains the estimated crack length after load variation. The bounds of this interval are derived from two extreme loading cases. The evolution of this interval for different prediction cycles $N_{p}$ is shown in Fig. 15 and in Fig. 16.

First of all, the gain of the detection algorithm and the reinitialization of the loading parameters values is highlighted in these figures. It can be seen that even if the loading parameters values after the load variation are uncertain, the interval that was derived from the interval $L$ still gives an accurate prediction of the future crack length. Moreover, the predictions improve as more data is available. Even if the plotted results give an idea about the efficiency of the proposed methodology, a more precise performance evaluation using metrics such as accuracy, precision and timeliness has been performed. The metrics were calculated for several reinitialization values of $\Delta \sigma_{a}$ and $\Delta P$ included in $L$. Thus, for each value of $\Delta \sigma_{a}$ and $\Delta P$ included in L, 100 RUL pdf computations were simulated to calculate the metrics. Then, the mean values and the standard deviation of each metrics in the predicted interval are given in Table 4.

The calculated metrics confirm that the proposed methodology gives satisfactory results in terms of accuracy, precision and timeliness despite the high uncertainty on the reinitialized values of the unknown loading parameters after load variation. Moreover, the obtained values show the usefulness of the timeliness metric. Indeed, we can see that the highest value of timeliness is at the prediction cycle $N_{p}=12.337 \times 10^{6}$. This is explained by the fact that a significant part of the predicted RUL have fallen after the actual RUL value. Thus, even if the accuracy is the highest one, the timeliness value must be taken into account carefully to optimize maintenance decisions and avoid catastrophic events. As for the case of the particle filter without the detection algorithm, the metrics could not even be calculated. Indeed, the particle filter did not manage to estimate the increase in crack length after the load variation. Therefore, the crack growth was so slow that the critical crack length could never be reached, making the calculation of the metrics impossible. 


\section{CONCLUSIONS}

In this work, fatigue crack growth prognosis in unidirectional fiber-reinforced composites using real data from fatigue tests on titanium matrix composites has been considered. First of all, the model that describes fatigue crack growth in such materials has been derived. For this purpose, the model that was previously developed in (Maire et al. 2000) has been simplified. Indeed, the problem in model-based prognosis is to make a compromise between a very accurate model that induces very high computational time, and a simplified model that will reduce computational time and effort. This simplified model was obtained by making assumptions on the physics-based model, and the resulting modeling uncertainties were represented with uncertain parameters and additive noise.

Then, using this nonlinear model and real crack growth data, two cases of fatigue crack prognosis in fiber-reinforced composite materials were treated. In a first study, the joint estimation of model parameters that depend on material in conjunction with the crack length was performed while assuming known value of the applied load. The performances of a particle filter and an extended Kalman filter (EKF) for the crack growth prognosis were compared using three prognosis metrics (accuracy, precision and timeliness). The results highlighted the high performance of the particle filter and the difficulty of the EKF to predict the future crack growth in presence of high nonlinearities. It was also shown that the more measurements are collected, the more the performance metrics of both filters were improved. However, the particle filter needed much less measurements to obtain a predicted RUL value close to the real one, which also proves the efficiency of the particle filter over the EKF in the studied crack growth prognosis.

In a second case study, a real crack growth dataset recorded under variable amplitude loading was used to perform fatigue crack growth prognosis in unidirectional fiber-reinforced composites under unknown applied load. A model-based on-line prognosis method that is able to estimate and forecast unknown loading parameters was proposed in this paper. A particle filter was used for the joint parameter-state estimation and a two-sided CUSUM algorithm was integrated to detect load variations. Indeed, it was noted that after an abrupt load variation, the particle filter had some 
difficulties to converge to the degradation state whose trajectory had suddenly changed. Therefore, the role of the CUSUM algorithm is to monitor the unknown loading parameters values, and to give an alert when a load variation is detected. Once the particle filter has received this alert, the estimated values of the unknown loading parameters are reinitialized in an interval that is chosen from a priori knowledge. The association of these two algorithms have enabled to keep the RUL predictions accurate even after load variation.

Finally, the simplified crack growth model that was proposed for fatigue crack growth prognosis in unidirectional fiber-reinforced composite materials can be validated. Indeed, even when considering that the absolute value of the closure pressure is equal to the applied load, the crack growth prognosis results were satisfactory. That is an important aspect in on-line prognosis because the computational time must be minimized.

In future work, the proposed prognosis method will be applied to fatigue crack growth data with more load variations, and other analytical models will be considered. Moreover, the problem of online filter tuning will be investigated.

\section{ACKNOWLEDGMENTS}

The authors would like to particularly acknowledge Pascal Paulmier and his colleagues of the Materials and Structures Department (DMAS) of ONERA for providing the datasets and valuable advice on the considered application. 


\section{APPENDIX I. REFERENCES}

Arulampalam, M. S., Maskell, S., Gordon, N., and Clapp, T. (2002). “A tutorial on particle filters for online nonlinear/non-Gaussian Bayesian tracking." IEEE Transactions on Signal Processing, 50(2), 174-188.

Awad, Z. K., Aravinthan, T., Zhuge, Y., and Gonzalez, F. (2012). "A review of optimization techniques used in the design of fibre composite structures for civil engineering applications." $M a$ terials \& Design, 33, 534-544.

Bakuckas, J. and Johnson, W. S. (1993). “Application of fiber bridging models to fatigue crack growth in unidirectional titanium matrix composites." Journal of Composites, Technology and Research, 15(3), 242-255.

Baraldi, P., Mangili, F., and Zio, E. (2013). "Investigation of uncertainty treatment capability of model-based and data-driven prognostic methods using simulated data." Reliability Engineering and System Safety, 112, 94-108.

Biagetti, T. (2004). "Automatic diagnostics and prognostics of energy conversion processes via knowledge-based systems.” Energy, 29(12-15), 2553-2572.

Blanke, M., Kinnaert, M., Lunze, J., Staroswiecki, M., and Schröder, J. (2006). Diagnosis and fault-tolerant control, Vol. 691. Springer.

Bueckner, H. (1970). "Novel principle for the computation of stress intensity factors." Zeitschrift fuer Angewandte Mathematik \& Mechanik, 50(9).

Chiachio, J., Chiachio, M., Saxena, A., Rus, G., and Goebel, K. (2013). “An energy-based prognostics framework to predict fatigue damage evolution in composites." Proceedings of the annual conference of the prognostics and health management society, 1(2), 363-371.

Corbetta, M., Sbarufatti, C., Manes, A., and Giglio, M. (2015). "Real-time prognosis of crack growth evolution using sequential monte carlo methods and statistical model parameters.' IEEE Transactions on Reliability, 64(2), 736-753.

Daigle, M. J. and Goebel, K. (2011). "A model-based prognostics approach applied to pneumatic valves." International journal of prognostics and health management, 2(2), 84-99. 
Daum, F. (2005). "Nonlinear filters: beyond the Kalman filter." Aerospace and Electronic Systems Magazine, 20(8), 57-69.

Davidson, D. (1992). "The micromechanics of fatigue crack growth at $25 \mathrm{c}$ in ti-6al-4v reinforced with scs-6 fibers." Metallurgical Transactions A, 23(3), 865-879.

Fan, J., Yung, K.-C., and Pecht, M. (2015). "Predicting long-term lumen maintenance life of led light sources using a particle filter-based prognostic approach.” Expert Systems with Applications, 42(5), 2411-2420.

Ghosn, L. J., Kantzos, P., and Telesman, J. (1992). "Modeling of crack bridging in a unidirectional metal matrix composite." International Journal of Fracture, 54(4), 345-357.

Gu, J., Barker, D., and Pecht, M. (2007). "Uncertainty assessment of prognostics of electronics subject to random vibration." AAAI fall symposium on artificial intelligence for prognostics,Arlington, VA, USA.

Johnson, W. S., Larsen, J. M., and Cox, B. (1996). Life prediction methodology for titanium matrix composites. Number 1253. ASTM International.

Kawai, M. and Itoh, N. (2014). "A failure-mode based anisomorphic constant life diagram for a unidirectional carbon/epoxy laminate under off-axis fatigue loading at room temperature." Journal of composite materials, 48(5), 571-592.

Kontoroupi, T. and Smyth, A. W. (2015). "Online noise identification for joint state and parameter estimation of nonlinear systems." ASCE-ASME Journal of Risk and Uncertainty in Engineering Systems, Part A: Civil Engineering, 2(3), B4015006.

Laseure, N., Schepens, I., Micone, N., and De Waele, W. (2015). "Effects of variable amplitude loading on fatigue life." Sustainable Construction and Design, 6(3).

Ling, Y. and Mahadevan, S. (2012). "Integration of structural health monitoring and fatigue damage prognosis.” Mechanical Systems and Signal Processing, 28, 89-104.

Liu, H., Yu, J., Zhang, P., and Li, X. (2009). "A review on fault prognostics in integrated health management." Proceedings of 9th International Conference on Electronic Measurement and Instruments, 4267-4270. 
Luo, J., Namburu, M., Pattipati, K., Qiao, L., Kawamoto, M., and Chigusa, S. (2003). "Modelbased prognostic techniques [maintenance applications]." Proceedings of IEEE AUTOTESTCON Conference, 330-340.

Maire, J., Levasseur, P., and Paulmier, P. (2000). "Multi-scale model of crack propagation in unidirectional metallic matrix composites.” ECF13, San Sebastian 2000.

McMeeking, R. and Evans, A. (1990). "Matrix fatigue cracking in fiber composites.” Mechanics of Materials, 9(3), 217-227.

Movchan, N. and Willis, J. (1996). "Critical load for a mode-1 crack reinforced by bridging fibres." The Quarterly Journal of Mechanics and Applied Mathematics, 49(4), 545-564.

Neerukatti, R. K., Liu, K. C., Kovvali, N., and Chattopadhyay, A. (2014). "Fatigue life prediction using hybrid prognosis for structural health monitoring." Journal of Aerospace Information Systems, 11(4), 211-232.

Orchard, M., Kacprzynski, G., Goebel, K., Saha, B., and Vachtsevanos, G. (2008). "Advances in uncertainty representation and management for particle filtering applied to prognostics." IEEE International Conference on Prognostics and Health Management, Denver, USA.

Orchard, M. E. and Vachtsevanos, G. J. (2007). “A particle filtering-based framework for real-time fault diagnosis and failure prognosis in a turbine engine." Mediterranean Conference on Control \& Automation, MED'07, IEEE.

Pais, M. J. and Kim, N. H. (2015). "Predicting fatigue crack growth under variable amplitude loadings with usage monitoring data.” Advances in Mechanical Engineering, 7(12), 1687814015619135 .

Robinson, E., Marzat, J., and Raïssi, T. (2016). "Model-based prognosis algorithms with uncertainty propagation: application to fatigue crack growth." 3rd Conference on Control and FaultTolerant Systems (SysTol'16), Barcelona, Spain, 443-450 (September).

Saha, B. and Goebel, K. (2008). "Uncertainty management for diagnostics and prognostics of batteries using bayesian techniques." IEEE Aerospace Conference, Big Sky, MT, USA, 1-8.

Saha, B., Goebel, K., Poll, S., and Christophersen, J. (2009). "Prognostics methods for battery 
health monitoring using a bayesian framework." IEEE Transactions on instrumentation and measurement, 58(2), 291-296.

Sankararaman, S. and Goebel, K. (2013). "Remaining useful life estimation in prognosis: An uncertainty propagation problem." AIAA Infotech at Aerospace (I at A) Conference, 1-8.

Si, X.-S., Wang, W., Hu, C.-H., and Zhou, D.-H. (2011). "Remaining useful life estimation A review on the statistical data driven approaches." European Journal of Operational Research, 213(1), 1-14.

Tada, H., Paris, P. C., and Irwin, G. R. (1973). "The stress analysis of cracks." Handbook, Del Research Corporation.

Thurner, T. (2015). "Real-time detection and measurement of cracks in fatigue test applications." AMA Conferences 2015-SENSOR 2015 and IRS2 2015, .

Wang, W., Hu, W., and Armstrong, N. (2017). "Fatigue crack prognosis using Bayesian probabilistic modelling." Mechanical Engineering Journal.

Zárate, B. A., Caicedo, J. M., Yu, J., and Ziehl, P. (2012). “Bayesian model updating and prognosis of fatigue crack growth.” Engineering Structures, 45, 53-61.

Zheng, D. and Ghonem, H. (1996). "High temperature/high frequency fatigue crack growth in titanium metal matrix composites." Life prediction methodology for titanium matrix composites, ASTM International.

Zio, E. and Di Maio, F. (2012). "Fatigue crack growth estimation by relevance vector machine." Expert Systems with Applications, 39(12), 10681-10692.

Zio, E. and Peloni, G. (2011). "Particle filtering prognostic estimation of the remaining useful life of nonlinear components." Reliability Engineering \& System Safety, 96(3), 403-409. 
TABLE 1: Distributions of random parameters for the estimation of $m$ and $C$

\begin{tabular}{ccccc}
\hline Parameter & $\log (C)$ & $m$ & $w$ & $v$ \\
\hline Variance & $\left(10^{-2}\right)^{2}$ & $\left(10^{-3}\right)^{2}$ & $\left(10^{-3}\right)^{2}$ & $\left(10^{-3}\right)^{2}$ \\
\hline
\end{tabular}


TABLE 2: Performance evaluation results for the estimation of $m$ and $C$

\begin{tabular}{ccccc}
\hline Prediction time & Method & Accuracy & Precision & Timeliness \\
\hline \multirow{2}{*}{$1.198 \times 10^{5}$} & PF & 0.88 & 0.72 & 0.77 \\
& EKF & 0.67 & 0.93 & 4.83 \\
\hline \multirow{2}{*}{$2.370 \times 10^{5}$} & PF & 0.95 & 0.82 & 0.17 \\
& EKF & 0.71 & 0.95 & 2.04 \\
\hline \multirow{2}{*}{$3.542 \times 10^{5}$} & PF & 0.96 & 0.85 & 0.08 \\
& EKF & 0.72 & 0.95 & 0.91 \\
\hline
\end{tabular}


TABLE 3: Distributions of random parameters for the estimation of $\Delta \sigma_{a}$ and $\Delta P$

\begin{tabular}{ccccc}
\hline Parameter & $\Delta \sigma_{a}$ & $\Delta P$ & $w$ & $v$ \\
\hline Variance & $(0.07)^{2}$ & $(0.07)^{2}$ & $\left(10^{-3}\right)^{2}$ & $\left(5 \times 10^{-3}\right)^{2}$ \\
\hline
\end{tabular}


TABLE 4: Performance evaluation results for the estimation of $\Delta \sigma_{a}$ and $\Delta P$

\begin{tabular}{cccc}
\hline Prediction time & Accuracy & Precision & Timeliness \\
\hline $11.058 \times 10^{6}$ & $0.59( \pm 0.04)$ & $0.62( \pm 0.02)$ & $4.56( \pm 1.05)$ \\
$11.197 \times 10^{6}$ & $0.65( \pm 0.04)$ & $0.75( \pm 0.02)$ & $2.74( \pm 0.73)$ \\
$11.335 \times 10^{6}$ & $0.81( \pm 0.08)$ & $0.86( \pm 0.04)$ & $0.92( \pm 0.46)$ \\
$12.337 \times 10^{6}$ & $0.87( \pm 0.15)$ & $0.59( \pm 0.07)$ & $207.12( \pm 486.04)$
\end{tabular}




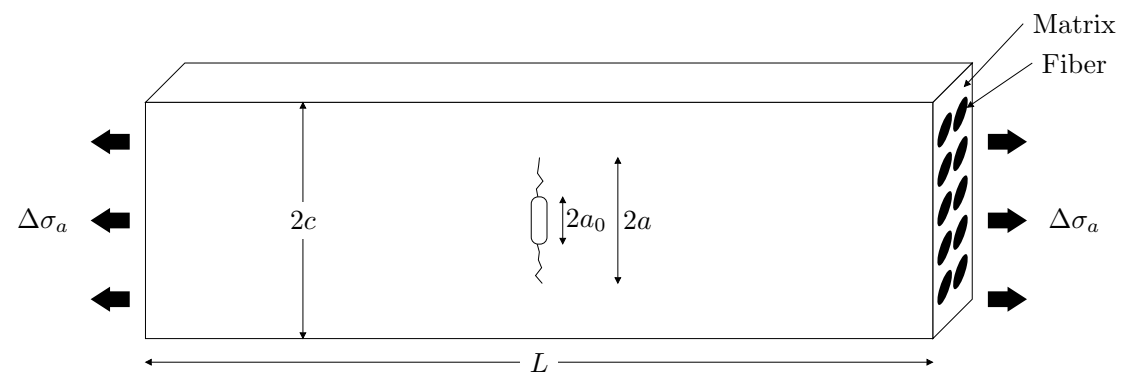

FIG. 1: Schematic of the specimen showing the dimensions and the loading axis parallel to fiber direction 


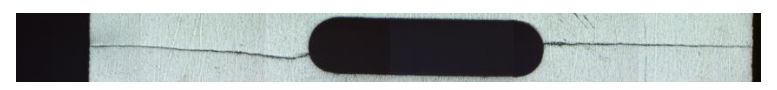

FIG. 2: Photography of the specimen with a crack perpendicular to fiber direction - With permission of ONERA/DMAS, Châtillon, France 


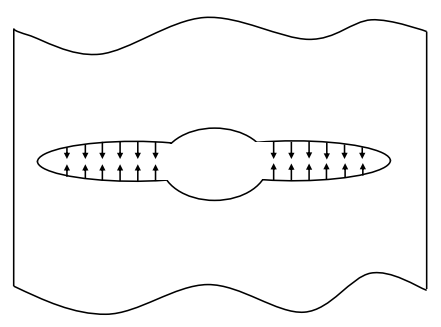

FIG. 3: The closure pressure in the crack wake 


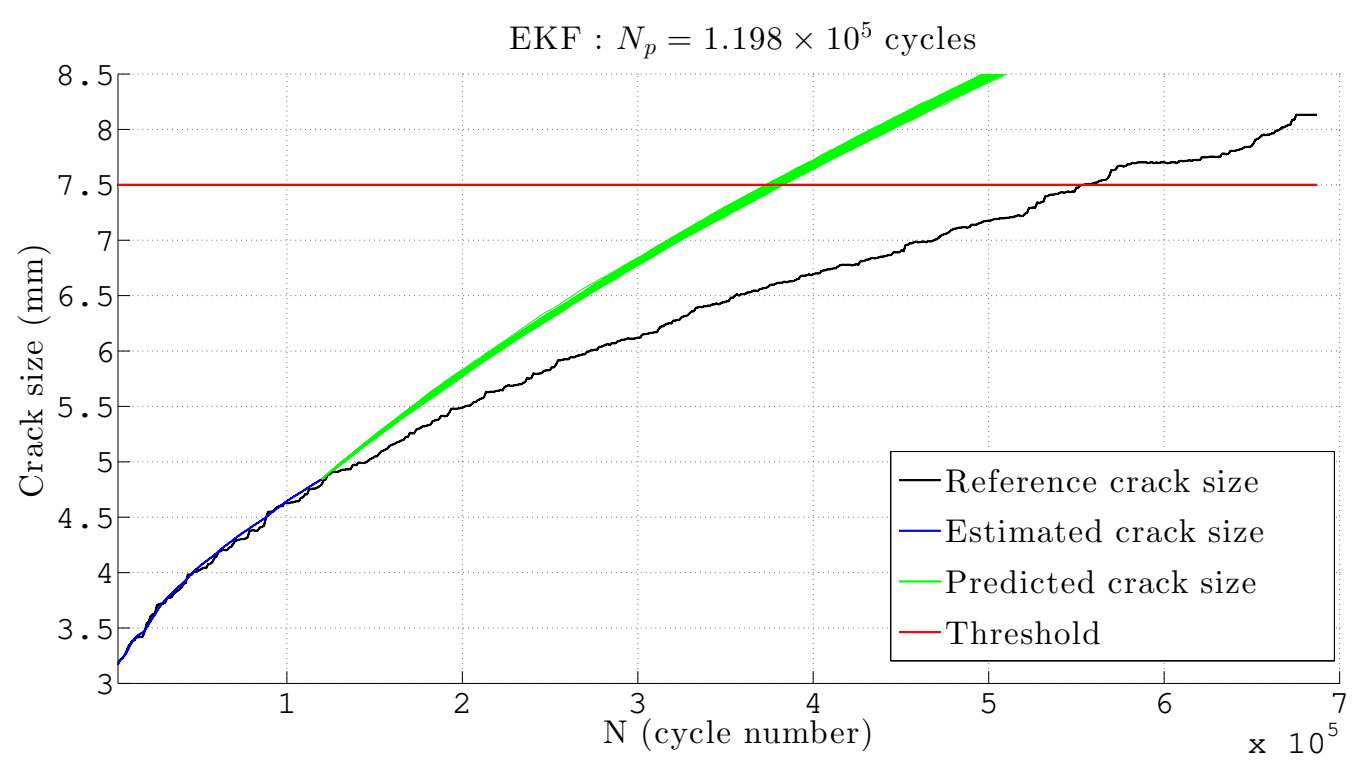

FIG. 4: Crack growth prognosis with the EKF at $N_{p}=1.198 \times 10^{5}$ cycles

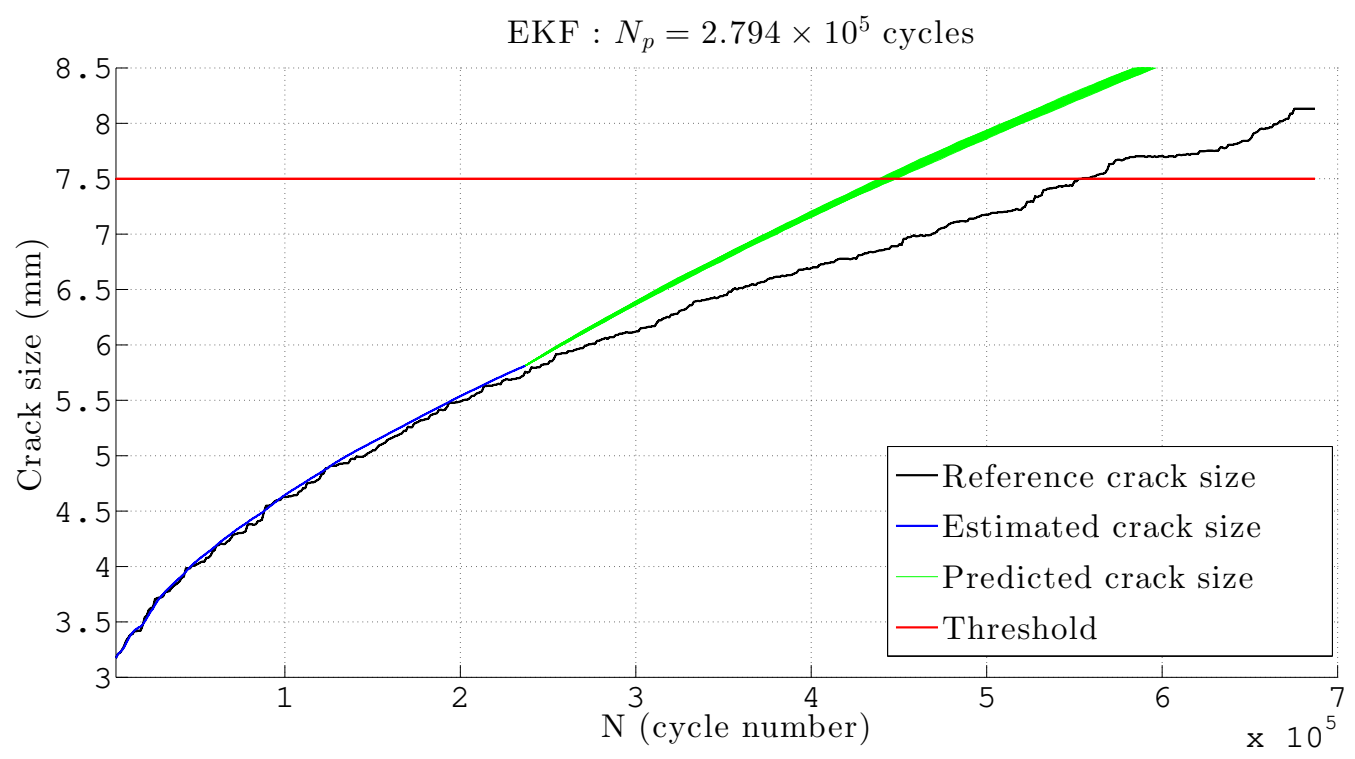

FIG. 5: Crack growth prognosis with the EKF at $N_{p}=2.794 \times 10^{5}$ cycles

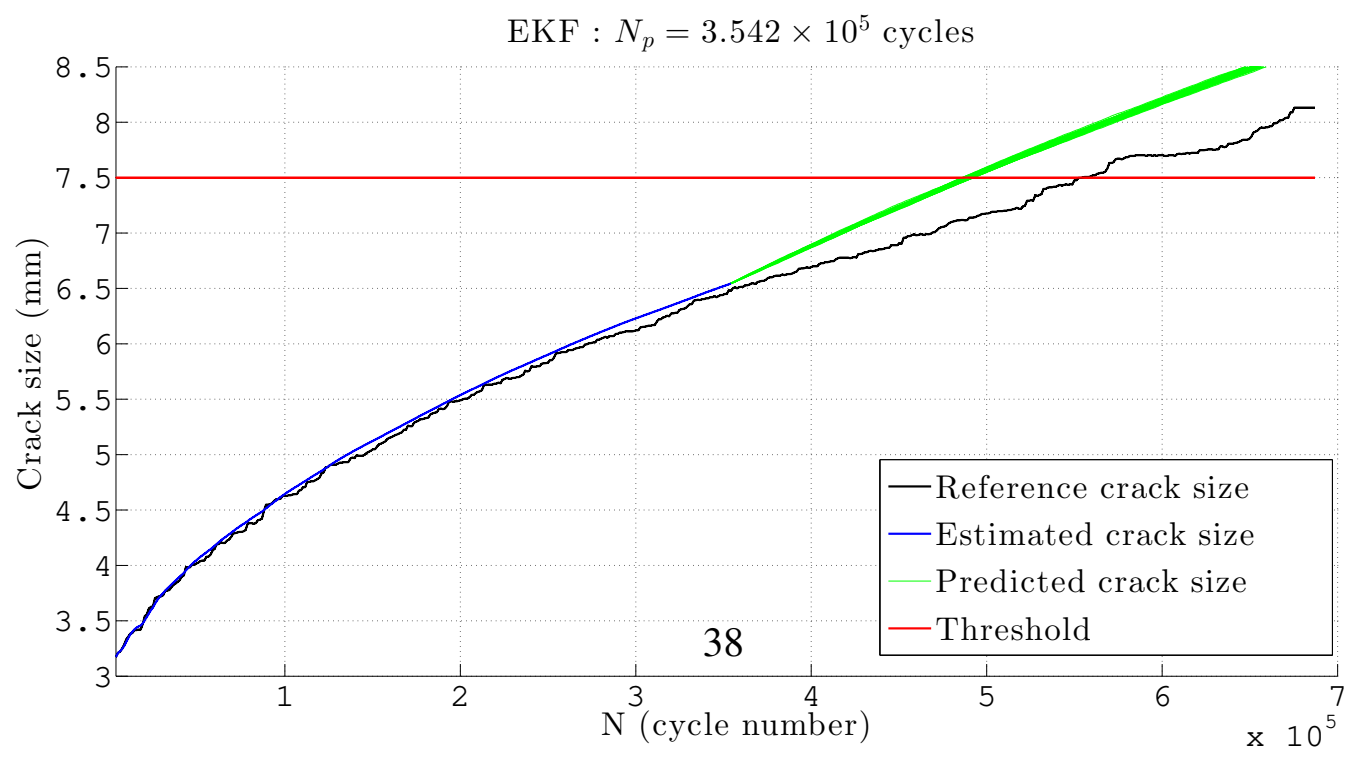




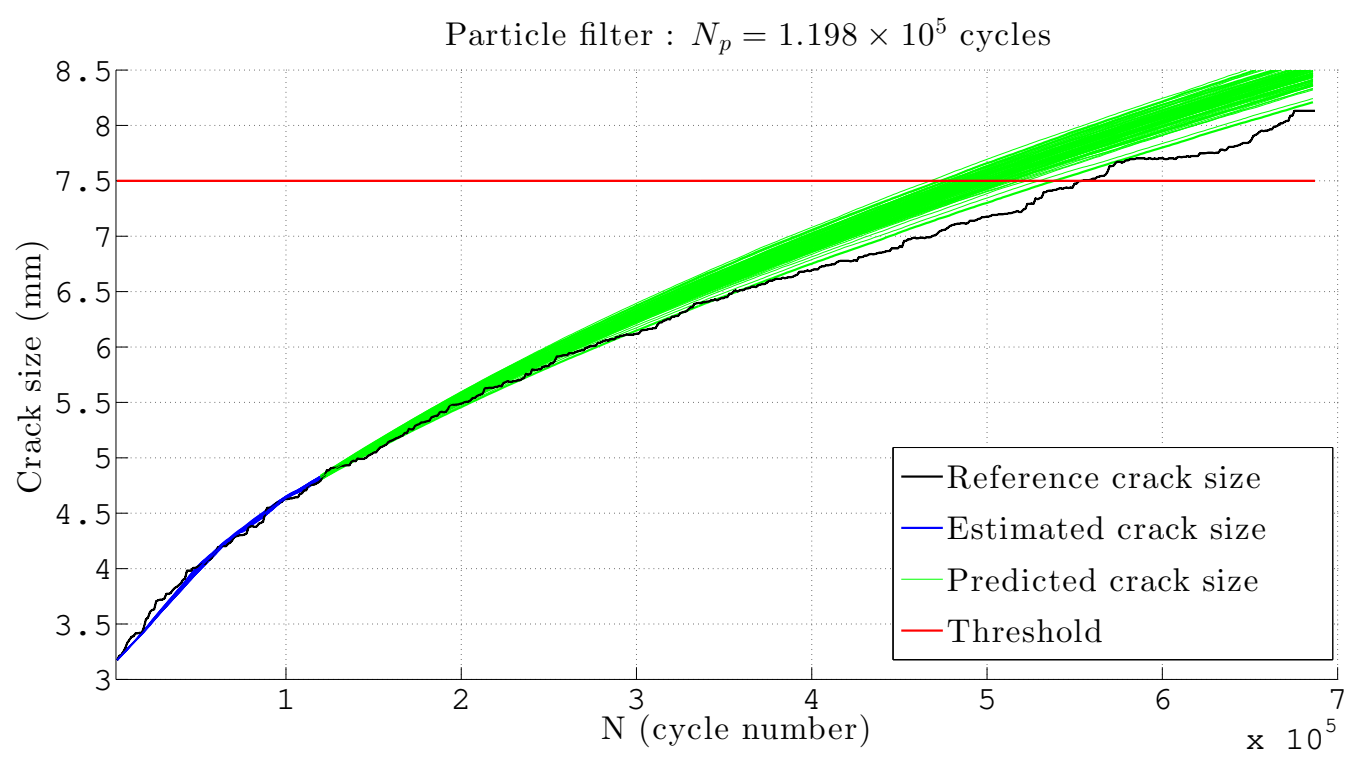

FIG. 7: Crack growth prognosis with the PF at $N_{p}=1.198 \times 10^{5}$ cycles

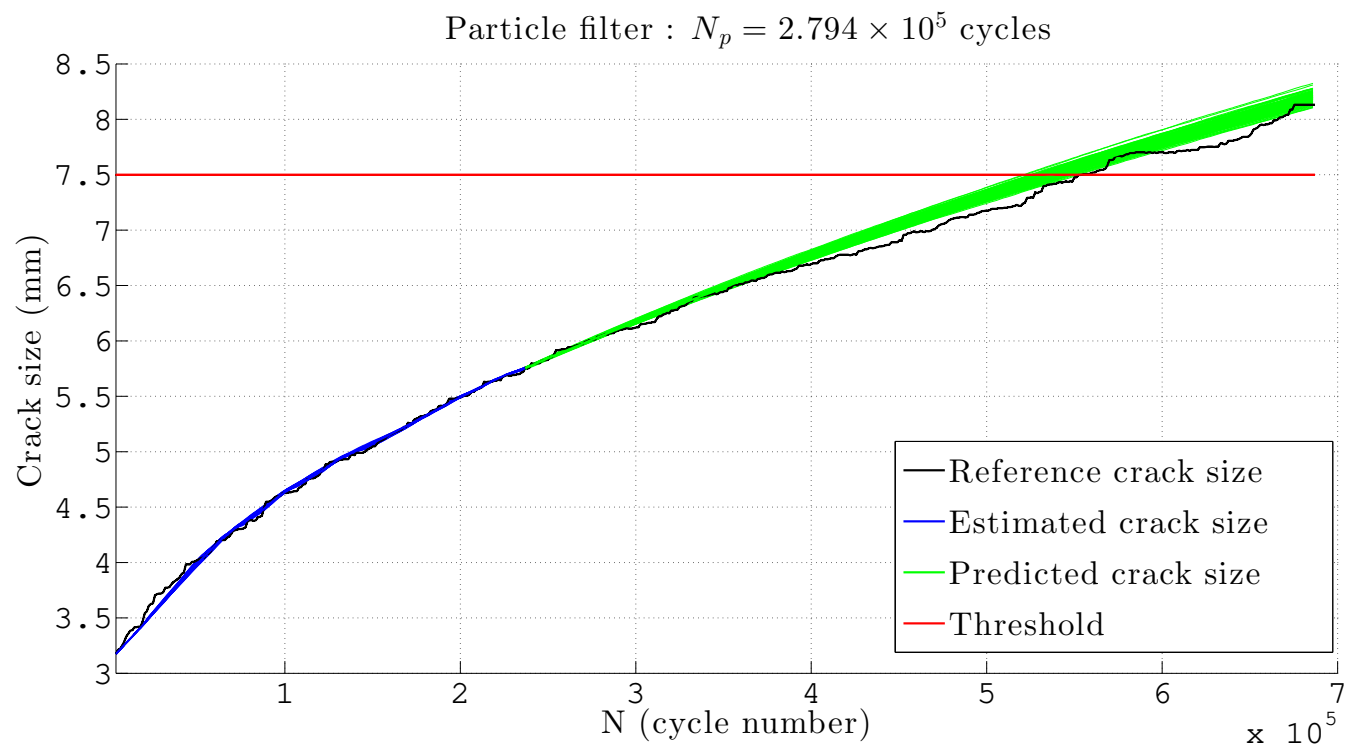

FIG. 8: Crack growth prognosis with the PF at $N_{p}=2.794 \times 10^{5}$ cycles

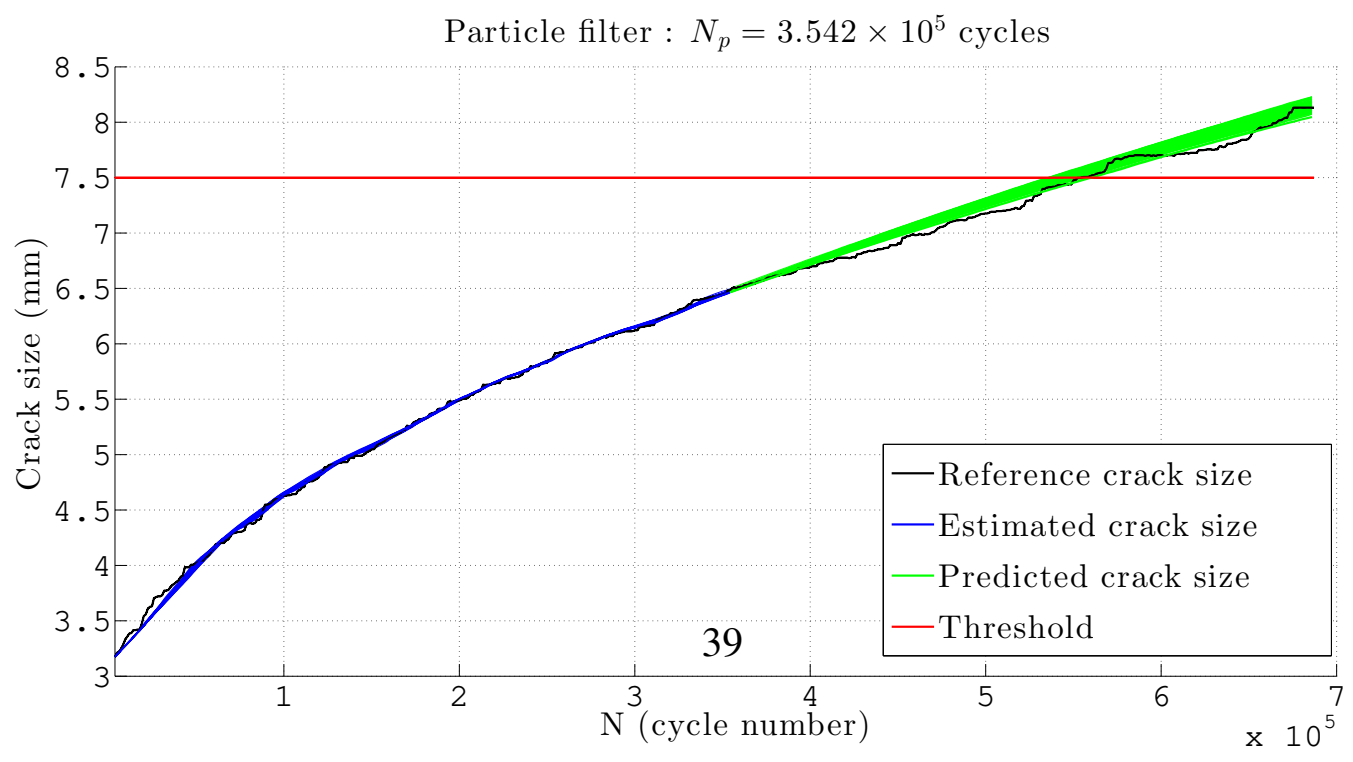




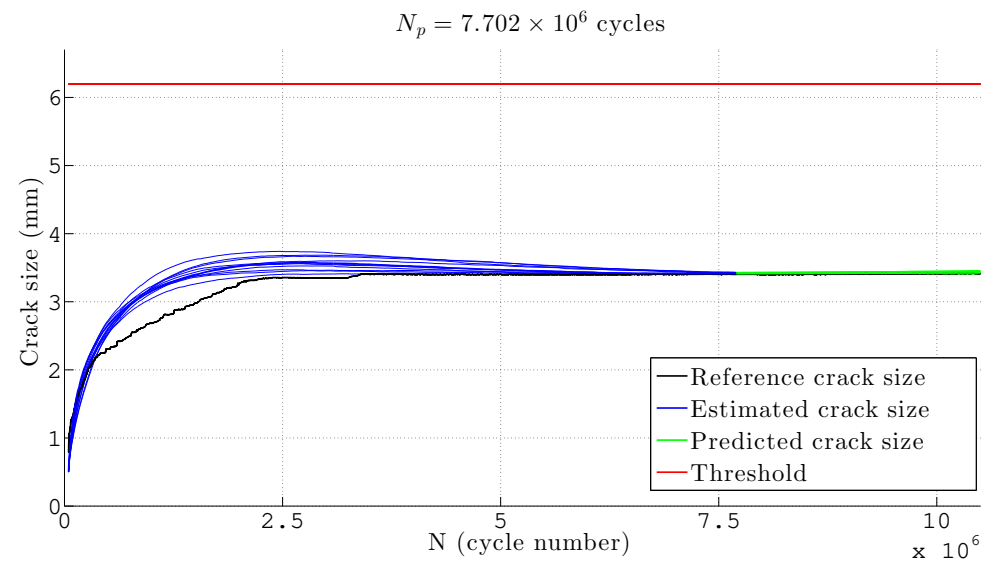

FIG. 10: Crack length evolution at $N_{p}=7.702 \times 10^{6}$ cycles 

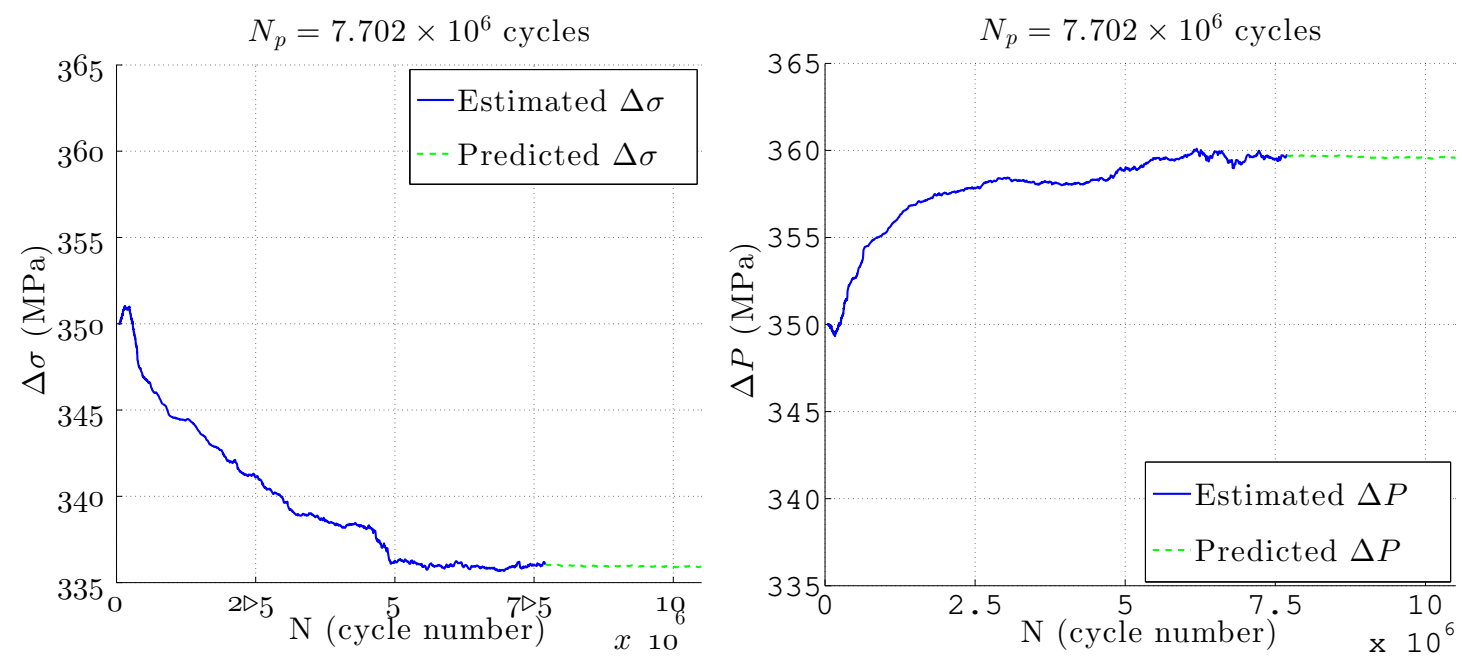

FIG. 11: Estimation of $\Delta P$ and $\Delta \sigma_{a}$ at $N_{p}=7.702 \times 10^{6}$ cycles 

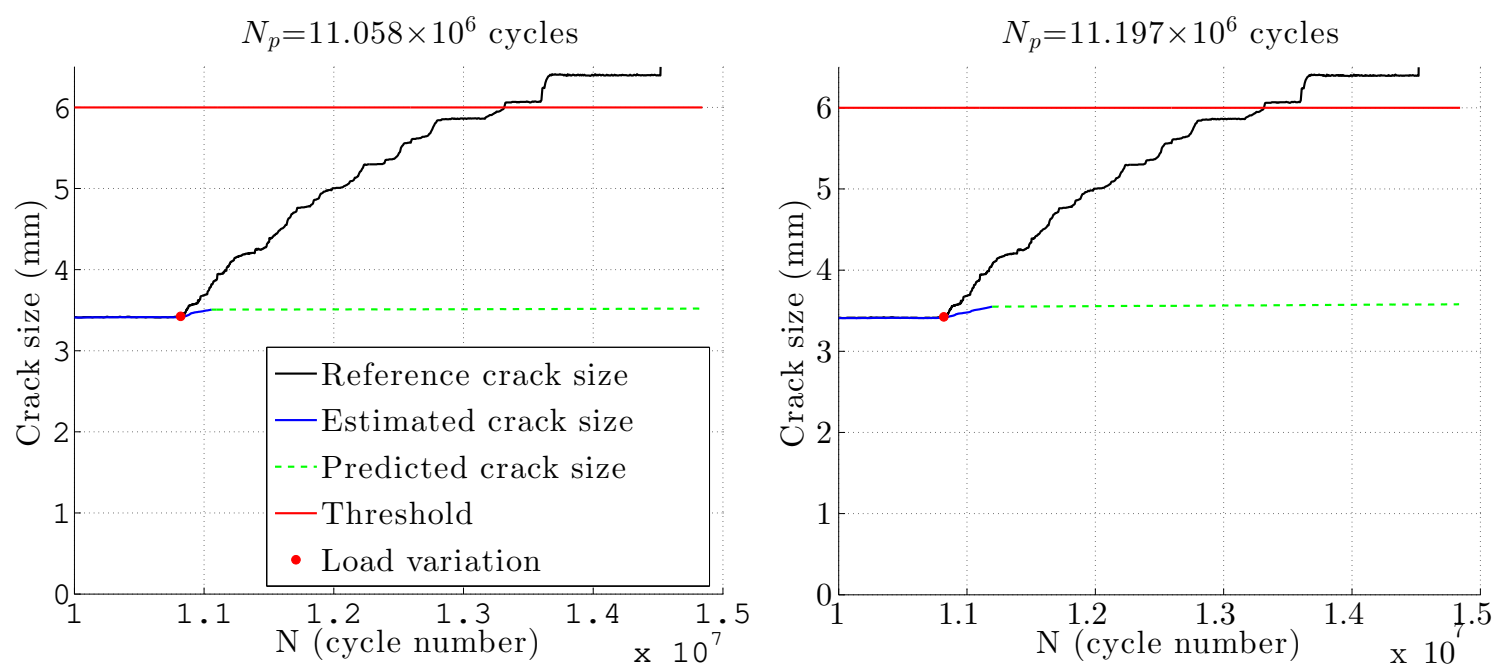

FIG. 12: Evolution of the crack length at different prediction cycles $N_{p}$ without the detection algorithm $\left(N_{p}=11.058 \times 10^{6}\right.$ cycles and $N_{p}=11.197 \times 10^{6}$ cycles $)$
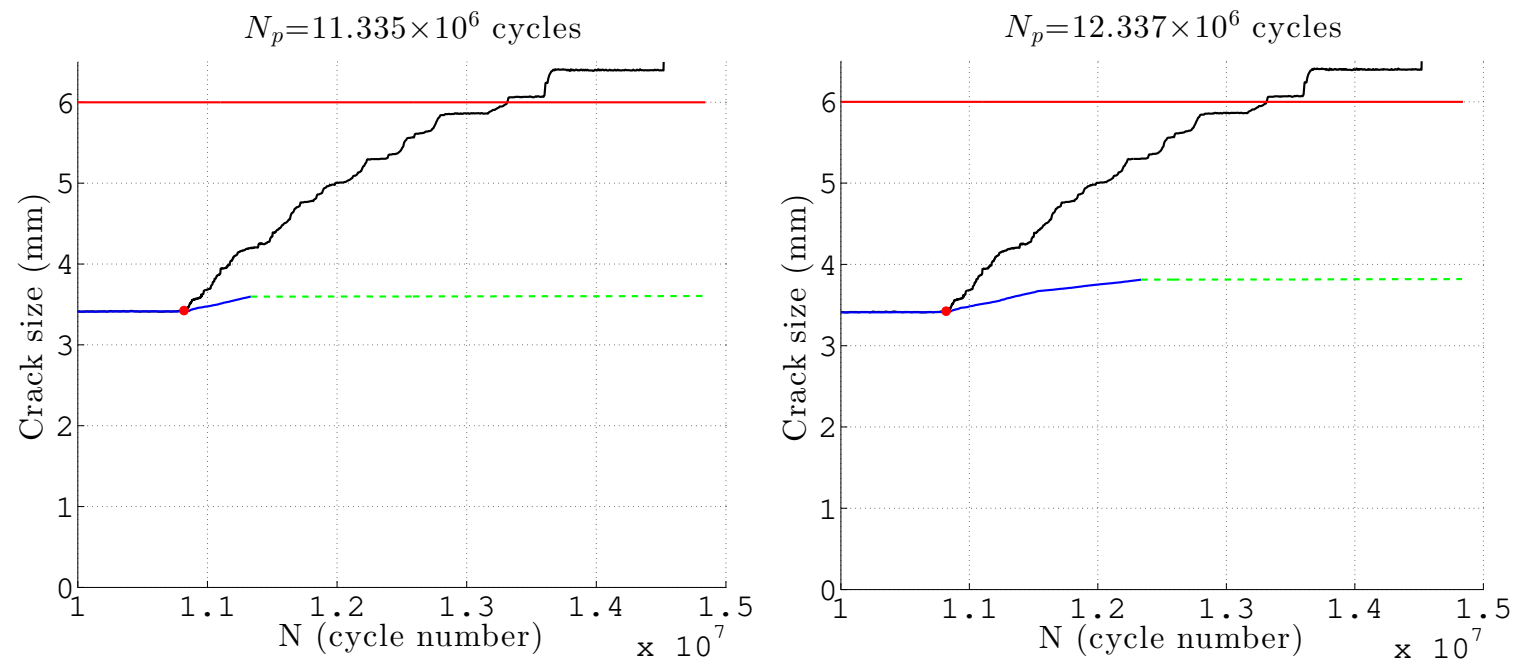

FIG. 13: Evolution of the crack length at different prediction cycles $N_{p}$ without the detection algorithm $\left(N_{p}=11.335 \times 10^{6}\right.$ cycles and $N_{p}=12.337 \times 10^{6}$ cycles $)$ 

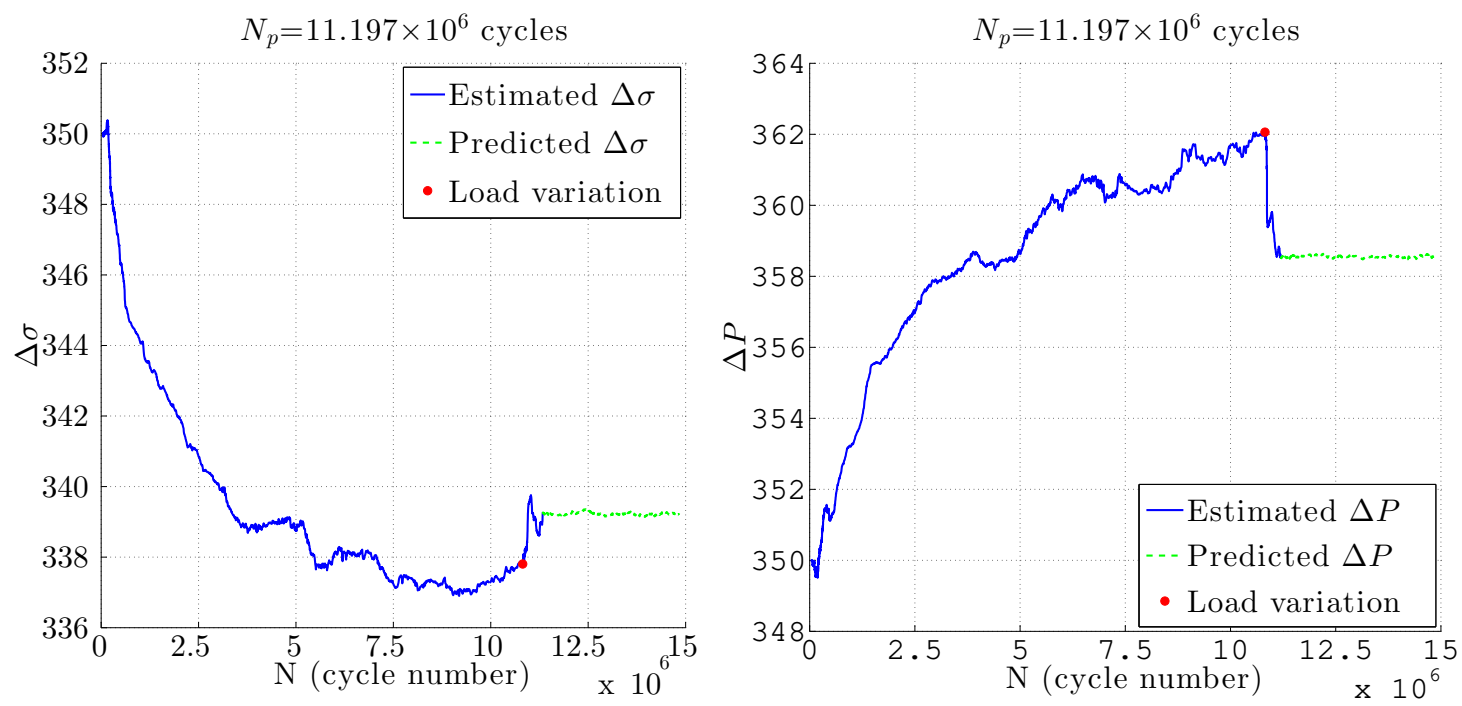

FIG. 14: Abrupt change in $\Delta \sigma_{a}$ and $\Delta P$ after load variation 

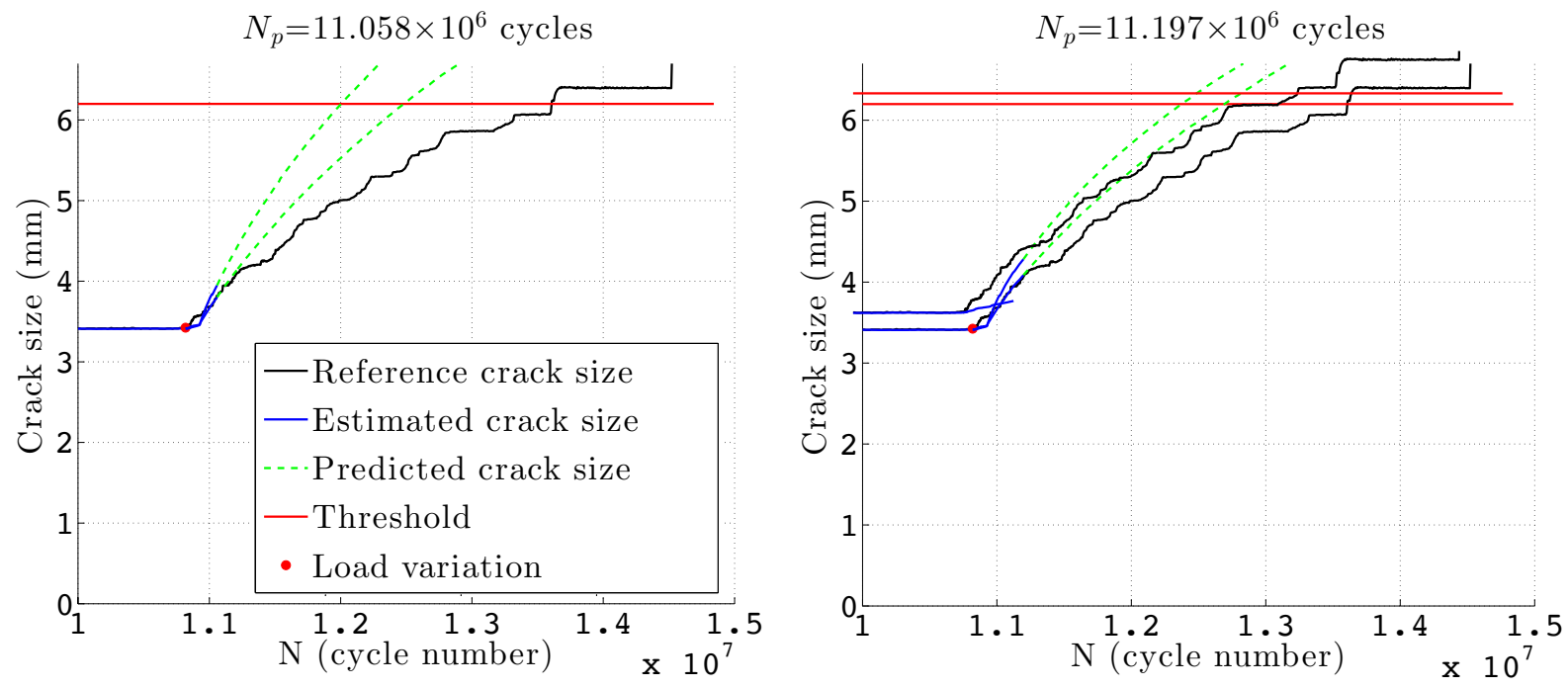

FIG. 15: Evolution of the crack length at different prediction cycles $N_{p}$ with the detection algorithm $\left(N_{p}=11.058 \times 10^{6}\right.$ cycles and $N_{p}=11.197 \times 10^{6}$ cycles $)$
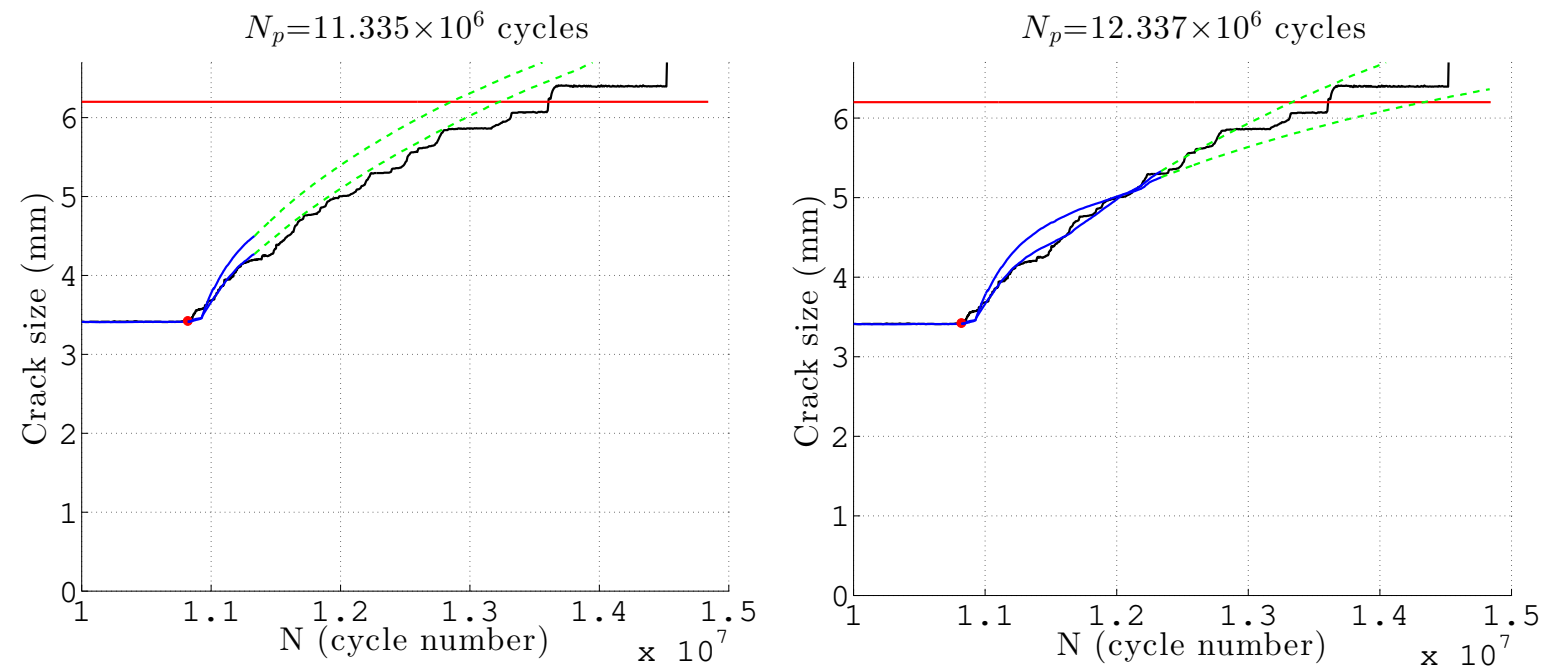

FIG. 16: Evolution of the crack length at different prediction cycles $N_{p}$ with the detection algorithm $\left(N_{p}=11.335 \times 10^{6}\right.$ cycles and $N_{p}=12.337 \times 10^{6}$ cycles $)$ 